\section{Multistep-type construction of fixed point for multivalued $\rho$-quasi-contractive-like maps in modular function spaces}

Multistep-type construction of fixed point...

\author{
Hudson Akewe and Hallowed Olaoluwa \\ Department of Mathematics, University of Lagos, Lagos, Nigeria
}

\begin{abstract}
Purpose - In this paper, the explicit multistep, explicit multistep-SP and implicit multistep iterative sequences are introduced in the context of modular function spaces and proven to converge to the fixed point of a multivalued map $T$ such that $P_{\rho}^{T}$, an associate multivalued map, is a $\rho$-contractive-like mapping.

Design/methodology/approach - The concepts of relative $\rho$-stability and weak $\rho$-stability are introduced, and conditions in which these multistep iterations are relatively $\rho$-stable, weakly $\rho$-stable and $\rho$-stable are established for the newly introduced strong $\rho$-quasi-contractive-like class of maps.

Findings - Noor type, Ishikawa type and Mann type iterative sequences are deduced as corollaries in this study.

Originality/value - The results obtained in this work are complementary to those proved in normed and metric spaces in the literature.
\end{abstract}

Keywords Multistep iterations, Modular function spaces, Strong $\rho$-contractions, Relative $\rho$-stability,

Weakly $\rho$-stability

Paper type Research paper

\section{Introduction and preliminary definitions}

Modular function spaces are well-known generalizations of both function and sequence variants of many important spaces such as Calderon-Lozanovskii, Kothe, Lebesgue, Lorentz, Musielak-Orlicz, Orlicz and Orlicz-Lorentz spaces. Their applications are also very useful. There is huge interest in quasi-contractive mappings in modular function spaces mainly because of the richness of structure of modular function spaces: apart from being F-spaces in a more general setting, they are equipped with modular equivalents of norm or metric notions and also endowed with convergence in submeasure. It is worthy to mention that modulartype conditions are far more natural as their assumptions can be easily verified than their corresponding metrics or norms, especially when related to fixed-point results and applications to integral-type operators. More so, there are some fixed-point results that can be proved only using the framework of modular function spaces. Thus, results in fixed-point theory in modular function spaces and those in normed and metric spaces are complementary (see, e.g. [1]). Different researchers have proved very useful fixed-points results in the context of modular function spaces (see [1-6] for details).

The following background definitions in $[1,3,7]$ are useful in proving the main results in this manuscript:

\section{JEL Classification - 47H10, 54H25}

(C) Hudson Akewe and Hallowed Olaoluwa. Published in Arab Journal of Mathematical Sciences. Published by Emerald Publishing Limited. This article is published under the Creative Commons Attribution (CCBY 4.0) licence. Anyone may reproduce, distribute, translate and create derivative works of this article (for both commercial and non-commercial purposes), subject to full attribution to the original publication and authors. The full terms of this licence may be seen at http://creativecommons. org/licences/by/4.0/legalcode
Received 23 July 2020 Revised 29 October 2020 Accepted 11 November 2020 
AJMS

27,2

190

Let $\Omega$ be a nonempty set and $\Sigma$ be a nontrivial $\sigma-$ algebra of subsets of $\Omega$. Let $\mathcal{P}$ be a $\delta$ - ring of subsets of $\Omega$ such that $E \cap A \in \mathcal{P}$ for any $E \in \mathcal{P}$ and $A \in \Sigma$. Assume there exists an increasing sequence $\left(K_{n}\right)_{n \in \mathrm{N}} \subset \mathcal{P}$ such that $\Omega=\cup_{n \in \mathrm{N}} K_{n}$.

Let $\mathcal{E}$ represent the linear space of all simple functions with supports from $\mathcal{P}$, that is, functions $s=\sum_{k=1}^{n} \alpha_{k} I_{A_{k}}$, where $\left(\alpha_{k}\right)_{k \in \mathrm{N}}$ is a sequence of real numbers, $\left(A_{k}\right)_{k \in \mathrm{N}}$ is a sequence of disjoint sets in $\mathcal{P}$ and $I_{A}$ represents the characteristic function of the set $A$ in $\Omega$.

Let $\mathscr{M}_{\infty}$ represent the space of all extended measurable functions, that is, all functions $f: \Omega \rightarrow[-\infty, \infty]$ such that there exists a sequence $\left(g_{n}\right) \subset \mathcal{E}$ satisfying $\left|g_{n}\right| \leq|f|$ and $g_{n}(\omega) \rightarrow f(\omega)$ for all $\omega \in \Omega$.

Definition 1.1. ([7]). Let $\rho: \mathscr{M}_{\infty} \rightarrow[0, \infty]$ be a nontrivial, convex and even function. $\rho$ is said to be a regular convex function pseudomodular if:

(1) $\rho(0)=0$;

(2) $\rho$ is monotone, that is, $|f| \leq|g|$ on $\Omega$ implies $\rho(f) \leq \rho(g)$, where $f, g \in \mathscr{M}_{\infty}$;

(3) $\rho$ is orthogonally subadditive, that is, $\rho\left(f I_{A \cup B}\right) \leq \rho\left(f I_{A}\right)+\rho\left(f I_{B}\right)$ for any $A, B \in \Omega$ such that $A \cap B \neq \phi$, with $f \in \mathscr{M}_{\infty}$;

(4) $\rho$ has Fatou's property, that is, $\left|f_{n}(\omega)\right| \uparrow|f(\omega)|$ for all $\omega \in \Omega$ implies $\rho\left(f_{n}\right) \uparrow \rho(f)$, where $f \in \mathscr{M}_{\infty}$;

(5) $\rho$ is order continuous in $\mathcal{E}$, that is, $\left(g_{n}\right) \subset \mathcal{E}$ and $\left|g_{n}(\omega)\right| \downarrow 0$ for all $\omega \in \Omega$ implies $\rho\left(g_{n}\right) \downarrow 0$.

Concepts similar to those in measure spaces are defined for function pseudomodular $\rho$ : a set $A \in \Sigma$ is said to be $\rho$-null if $\rho\left(f I_{A}\right)=0 \forall f \in \mathcal{E}$; a property is said to hold $\rho$-almost everywhere ( $\rho$-a.e.) on $\Sigma$ if the set for which it does not hold is $\rho$-null.

The following set is defined:

$$
\mathscr{M}(\Omega, \Sigma, \mathcal{P}, \rho)=\left\{f \in \mathscr{M}_{\infty}:|f|<\infty \rho-\text { a.e. }\right\},
$$

where each $f \in \mathscr{M}_{\infty}$ is actually an equivalence class of functions equal $\rho$-a.e. We will write $\mathscr{M}$ instead of $\mathscr{M}(\Omega, \Sigma, \mathcal{P}, \rho)$ when no confusion arises.

Definition 1.2. ([1]). Let $\rho$ be a regular function pseudomodular.

(1) $\rho$ is said to be a regular function modular if $\rho(f)=0$ implies $f=0 \rho$-a.e.

(2) $\rho$ is said to be a regular function semimodular if $\rho(\alpha f)=0$ for every $\alpha>0$ implies $f=0 \rho$-a.e.

A regular convex function modular $\rho$ satisfies the following properties (see [3])

(1) $\rho(f)=0$ if $f=0 \rho$-a.e.

(2) $\rho(\alpha f)=\rho(f)$ for every scalar $\alpha$ such that $|\alpha|=1$, where $f \in \mathscr{M}$.

(3) $\rho(\alpha f+\beta g) \leq \alpha \rho(f)+\beta \rho(g)$ if $\alpha+\beta=1, \alpha, \beta \geq 0$ and $f, g \in \mathscr{M}$.

The class of all nonzero regular convex function modulars on $\Omega$ is denoted by $\mathfrak{R}$.

Definition 1.3. ([7]). A convex function modular $\rho$ defines the modular function space $L_{\rho}$ as

$$
L_{\rho}=\{f \in \mathscr{M}: \rho(\lambda f) \rightarrow 0 \text { as } \lambda \rightarrow 0\} .
$$


$L_{\rho}$ is a normed linear space with respect to

$$
\left|f_{\rho}\right|=\inf \left\{\alpha>0: \rho\left(\frac{f}{\alpha}\right) \leq 1\right\}
$$

which is known as the Luxemburg norm.

Definition 1.4. ([7]). Let $L_{\rho}$ be a modular space. The sequence $\left\{f_{n}\right\} \subset L_{\rho}$ is called:

(1) $\rho$-convergent to $f \in L_{\rho}$ if $\rho\left(f_{n}-f\right) \rightarrow 0$ as $n \rightarrow \infty$;

(2) $\rho$-Cauchy, if $\rho\left(f_{n}-f_{m}\right) \rightarrow 0$ as $n, m \rightarrow \infty$.

Remark 1.1. $\rho$-convergent sequence implies $\rho$-Cauchy sequence if and only if $\rho$ satisfies the $\Delta_{2}$ - condition given in the definition below. However, $\rho$ does not satisfy the triangle inequality.

Definition 1.5. ([7]). A nonzero regular convex function $\rho$ is said to satisfy the $\Delta_{2}-$ condition, if $\sup _{n \geq 1} \rho\left(2 f_{n}, D_{k}\right) \rightarrow 0$ as $k \rightarrow \infty$ whenever $\left\{D_{k}\right\} \downarrow \emptyset /$ and $\sup _{n \geq 1} \rho\left(f_{n}, D_{k}\right) \rightarrow 0$ as $k \rightarrow \infty$.

Definition 1.6. ([7]). Let $L_{\rho}$ be a modular space and $D \subset L_{\rho}$.

The $\rho$-distance from $f \in L_{\rho}$ to the set $D$ is given by:

$$
\operatorname{dist}_{\rho}(f, D)=\inf \{\rho(f-h): h \in D\} .
$$

A subset $D \subset L_{\rho}$ is called:

(1) $\rho$-closed if the $\rho$ - limit of a $\rho$-convergent sequence of $D$ always belongs to $D$;

(2) $\rho$-a.e. closed if the $\rho-a . e$. limit of a $\rho-a$.e. convergent sequence of $D$ always belongs to $D$;

(3) $\rho$-compact if every sequence in $D$ has a $\rho$-convergent subsequence in $D$;

(4) $\rho$-a.e. compact if every sequence in $D$ has a $\rho-a . e$. convergent subsequence in $D$;

(5) $\rho$-bounded if $\operatorname{diam}_{\rho}(D)=\sup \{\rho(f-g): f, g \in D\}<\infty$.

(6) $\rho$-proximal if for each $f \in L_{\rho}$ there exists an element $g \in D$ such that $\rho(f-g)=\operatorname{dist}_{\rho}(f, D)$.

The family of nonempty $\rho$-bounded $\rho$-proximal subsets of $D$ is denoted by $P_{\rho}(D)$, the family of nonempty $\rho$-closed $\rho$-bounded subsets of $D$ by $C_{\rho}(D)$ and the family of $\rho$-compact subsets of $D$ by $K_{\rho}(D)$.

Definition 1.7. ([7]). Let $L_{\rho}$ be a modular space. A function $f \in L_{\rho}$ is called a fixed point of a multivalued mapping $T: L_{\rho} \rightarrow P_{\rho}(D)$ if $f \in T f$. The set of all fixed points of $T$ is represented by $F_{\rho}(T)$ so that:

$$
F_{\rho}(T)=\left\{f \in L_{\rho}: f \in T f\right\} .
$$

The following set is also defined:

$$
P_{\rho}^{T}(f)=\left\{g \in T f: \rho(f-g)=\operatorname{dist}_{\rho}(f, T f)\right\} .
$$

Zamfirescu [8] in 1972 proved the following theorem as a generalization of the Banach fixedpoint theorem: 
AJMS

27,2

192

Theorem 1.1. ([8]). Let $X$ be a complete metric space and $T: X \rightarrow X$ a Zamfirescu operator satisfying:

$$
d(T x, T y) \leq h \max \left\{d(x, y), \frac{d(x, T x)+d(y, T y)}{2}, \frac{d(x, T y)+d(y, T x)]}{2}\right\},
$$

where $0 \leq h<1$. Then, $T$ has a unique fixed point and the Picard iteration converges to $p$ for any $x_{0} \in X$.

Observe that in a Banach space setting, condition (1.1) implies

$$
\|T x-T y\| \leq \delta\|x-y\|+2 \delta\|x-T x\|, \delta=\max \left\{h, \frac{h}{2-h}\right\} \in[0,1)
$$

Osilike [9] used the following contractive definition: for each $x, y \in X$, there exist $\delta \in[0,1)$ and $L \geq 0$ such that

$$
\|T x-T y\| \leq \delta\|x-y\|+L\|x-T x\| .
$$

Imoru and Olatinwo [10] proved some stability results using the following general contractive definition: for each $x, y \in X$, there exist $\delta \in[0,1)$ and a monotone increasing function $\varphi: \mathbb{R}^{+} \rightarrow \mathbb{R}^{+}$with $\varphi(0)=0$ such that

$$
\|T x-T y\| \leq \delta\|x-y\|+\varphi(\|x-T x\|) .
$$

Observe that (1.4) generalizes (1.3) and (1.2). The map $T$ considered in (1.2)-(1.4) is singlevalued. Now, we state the generalizations of (1.2)-(1.4) to multivalued mappings, as conformed to literature. (e.g. see [7]).

Let $H_{\rho}(\cdot, \cdot)$ be the $\rho$-Hausdorff distance on the family $C_{\rho}\left(L_{\rho}\right)$ of nonempty $\rho$-closed $\rho$-bounded subsets of $L_{\rho}$, that is,

$$
H_{\rho}(A, B)=\max \left\{\sup _{f \in A} \operatorname{dist}_{\rho}(f, B), \sup _{g \in B} \operatorname{dist}_{\rho}(g, A)\right\}, A, B \in C_{\rho}\left(L_{\rho}\right) .
$$

A multivalued map $T: D \rightarrow C_{\rho}\left(L_{\rho}\right)$ is said to be a:

(1) $\rho$-contraction mapping if there exists a constant $\delta \in[0,1)$ such that

$$
H_{\rho}(T f, T g) \leq \delta \rho(f-g), \forall f, g \in D .
$$

(2) $\rho$-Zamfirescu mapping if

$$
H_{\rho}(T f, T g) \leq \delta \rho(f-g)+2 \delta \rho(h-f), \forall f, g \in D \forall h \in T f .
$$

(3) $\rho$-quasi-contractive mapping if

$$
H_{\rho}(T f, T g) \leq \delta \rho(f-g)+L \rho(h-f), \forall f, g \in D \forall h \in T f, L \geq 0 .
$$

(4) $\rho$-quasi-contractive-like mapping if

$$
H_{\rho}(T f, T g) \leq \delta \rho(f-g)+\varphi(\rho(h-f)), \forall f, g \in D \forall h \in T f .
$$

where $\varphi: \mathbb{R}^{+} \rightarrow \mathbb{R}^{+}$is a monotone increasing function with $\varphi(0)=0$.

Convergence and stability of fixed-point iterative sequences for single mapping $T$ are two very vital concepts in fixed-point theory and applications. Some of the results of colossal value in this work are those in [9-20]. Rhoades and Soltuz [21] introduced the multistep iteration and proved its equivalence with Mann and Ishikawa iterations. Olaleru and Akewe [22] proved convergence of multistep iteration for a pair of mappings $(S, T)$ 
We now introduce the following iterative sequences in the framework of modular function spaces and use them to prove new fixed-point theorems.

Let $T: D \rightarrow P_{\rho}(D)$ be a multivalued mapping.

The explicit multistep iterative sequence $\left\{f_{n}\right\}_{n=0}^{\infty} \subset D$ is defined by:

$$
\left\{\begin{array}{cccc}
f_{0} & \in & D \\
f_{n+1} & = & \left(1-\alpha_{n}\right) f_{n}+\alpha_{n} v_{n}^{1}, & \\
g_{n}^{i} & = & \left(1-\beta_{n}^{i}\right) f_{n}+\beta_{n}^{i} v_{n}^{i+1}, & i=1,2, \ldots, k-2 \\
g_{n}^{k-1} & = & \left(1-\beta_{n}^{k-1}\right) f_{n}+\beta_{n}^{k-1} u_{n}, & n=0,1,2, \ldots
\end{array}\right.
$$

where $u_{n} \in P_{\rho}^{T}\left(f_{n}\right), v_{n}^{i} \in P_{\rho}^{T}\left(g_{n}^{i}\right), i=1,2, \ldots, k-1$, and the sequences $\left\{\alpha_{n}\right\}_{n=0}^{\infty}$ and $\left\{\beta_{n}^{i}\right\}_{n=0}^{\infty}$, $i=1,2, \ldots, k-1$, are in $[0,1)$ such that $\sum_{n=0}^{\infty} \alpha_{n}=\infty$.

The explicit Noor iterative sequence $\left\{f_{n}\right\}_{n=0}^{\infty} \subset D$ is defined by:

$$
\left\{\begin{array}{ccc}
f_{0} & \in & D \\
f_{n+1}= & \left(1-\alpha_{n}\right) f_{n}+\alpha_{n} v_{n}^{1}, \\
g_{n}^{1}= & \left(1-\beta_{n}^{1}\right) f_{n}+\beta_{n}^{1} v_{n}^{2}, \\
g_{n}^{2}= & \left(1-\beta_{n}^{2}\right) f_{n}+\beta_{n}^{2} u_{n}, n=0,1,2, \ldots
\end{array}\right.
$$

where $u_{n} \in P_{\rho}^{T}\left(f_{n}\right), v_{n}^{1} \in P_{\rho}^{T}\left(g_{n}^{1}\right), v_{n}^{2} \in P_{\rho}^{T}\left(g_{n}^{2}\right)$, and the sequences $\left\{\alpha_{n}\right\}_{n=0}^{\infty},\left\{\beta_{n}^{1}\right\}_{n=0}^{\infty}$ and $\left\{\beta_{n}^{2}\right\}_{n=0}^{\infty}$ are in $[0,1)$ such that $\sum_{n=0}^{\infty} \alpha_{n}=\infty$.

The explicit Ishikawa iterative sequence $\left\{f_{n}\right\}_{n=0}^{\infty} \subset D$ is defined by:

$$
\left\{\begin{array}{ccc}
f_{0} & \in & D \\
f_{n+1} & = & \left(1-\alpha_{n}\right) f_{n}+\alpha_{n} v_{n}^{1}, \\
g_{n}^{1} & = & \left(1-\beta_{n}^{1}\right) f_{n}+\beta_{n}^{1} u_{n}, n=0,1,2, \ldots
\end{array}\right.
$$

where $u_{n} \in P_{\rho}^{T}\left(f_{n}\right), v_{n}^{1} \in P_{\rho}^{T}\left(g_{n}^{1}\right)$, and the sequences $\left\{\alpha_{n}\right\}_{n=0}^{\infty}$ and $\left\{\beta_{n}^{1}\right\}_{n=0}^{\infty}$ are in $[0,1)$ such that $\sum_{n=0}^{\infty} \alpha_{n}=\infty$.

The explicit Mann iterative sequence $\left\{f_{n}\right\}_{n=0}^{\infty} \subset D$ is defined by:

$$
\left\{\begin{array}{ccc}
f_{0} & \in & D \\
f_{n+1} & = & \left(1-\alpha_{n}\right) f_{n}+\alpha_{n} u_{n}, n=0,1,2, \ldots
\end{array}\right.
$$

where $u_{n} \in P_{\rho}^{T}\left(f_{n}\right),\left\{\alpha_{n}\right\}_{n=0}^{\infty} \subset[0,1)$ and $\sum_{n=0}^{\infty} \alpha_{n}=\infty$.

The explicit multistep-SP iterative sequence $\left\{f_{n}\right\}_{n=0}^{\infty} \subset D$ is defined by:

$$
\left\{\begin{array}{cccc}
f_{0} & \in & D \\
f_{n+1} & = & \left(1-\alpha_{n}\right) g_{n}^{1}+\alpha_{n} v_{n}^{1} & \\
g_{n}^{i} & = & \left(1-\beta_{n}^{i}\right) g_{n}^{i+1}+\beta_{n}^{i} v_{n}^{i+1}, & i=1,2, \ldots, k-2 \\
g_{n}^{k-1} & = & \left(1-\beta_{n}^{k-1}\right) f_{n}+\beta_{n}^{k-1} u_{n}, & n=0,1,2, \ldots
\end{array}\right.
$$

where $u_{n} \in P_{\rho}^{T}\left(f_{n}\right), v_{n}^{i} \in P_{\rho}^{T}\left(g_{n}^{i}\right), i=1,2, \ldots, k-1$, and the sequences $\left\{\alpha_{n}\right\}_{n=0}^{\infty}$ and $\left\{\beta_{n}^{i}\right\}_{n=0}^{\infty}$, $i=1,2, \ldots, k-1$, are in $[0,1)$ such that $\sum_{n=0}^{\infty} \alpha_{n}=\infty$. 


$$
\left\{\begin{array}{ccc}
f_{0} & \in & D \\
f_{n+1}= & \left(1-\alpha_{n}\right) g_{n}^{1}+\alpha_{n} v_{n}^{1}, \\
g_{n}^{1}= & \left(1-\beta_{n}^{1}\right) g_{n}^{2}+\beta_{n}^{1} v_{n}^{2}, \\
g_{n}^{2}= & \left(1-\beta_{n}^{2}\right) f_{n}+\beta_{n}^{2} u_{n}, n=0,1,2, \ldots
\end{array}\right.
$$

where $u_{n} \in P_{\rho}^{T}\left(f_{n}\right), v_{n}^{1} \in P_{\rho}^{T}\left(g_{n}^{1}\right), v_{n}^{2} \in P_{\rho}^{T}\left(g_{n}^{2}\right)$, and the sequences $\left\{\alpha_{n}\right\}_{n=0}^{\infty},\left\{\beta_{n}^{1}\right\}_{n=0}^{\infty}$, and $\left\{\beta_{n}^{2}\right\}_{n=0}^{\infty}$ are in $[0,1)$ such that $\sum_{0}^{\infty} \alpha_{n}=\infty$.

The implicit multistep iterative sequence $\left\{f_{n}\right\}_{n=0}^{\infty} \subset D$ is defined by:

$$
\left\{\begin{array}{cccc}
f_{0} & \in & D \\
f_{n+1} & = & \left(1-\alpha_{n}\right) f_{n}^{1}+\alpha_{n} u_{n+1}, & \\
f_{n}^{i} & = & \left(1-\beta_{n}^{i}\right) f_{n}^{i+1}+\beta_{n}^{i} u_{n}^{i}, & i=1,2, \ldots, k-2 \\
f_{n}^{k-1} & = & \left(1-\beta_{n}^{k-1}\right) f_{n}+\beta_{n}^{k-1} u_{n}^{k-1}, & n=0,1,2, \ldots
\end{array}\right.
$$

where $u_{n+1} \in P_{\rho}^{T}\left(f_{n}\right), u_{n}^{i} \in P_{\rho}^{T}\left(f_{n}^{i}\right), i=1,2, \ldots, k-1$, and the sequences $\left\{\alpha_{n}\right\}_{n=0}^{\infty}$ and $\left\{\beta_{n}^{i}\right\}_{n=0}^{\infty}, i=1,2, \ldots, k-1$, are in $[0,1)$ such that $\sum_{n=0}^{\infty} \alpha_{n}=\infty$.

It should be noted that the implicit multistep iterative sequence exists if and only if $T$ satisfies the property (I) as follows:

$$
(I): \forall h \in D \quad \forall \beta \in(0,1) \exists f \in D \quad \exists g \in P_{\rho}^{T}(f): \quad f=(1-\beta) h+\beta g .
$$

The implicit Noor iterative sequence $\left\{f_{n}\right\}_{n=0}^{\infty} \subset D$ is defined by:

$$
\left\{\begin{array}{ccc}
f_{0} & \in & D \\
f_{n+1} & = & \left(1-\alpha_{n}\right) f_{n}^{1}+\alpha_{n} u_{n+1}, \\
f_{n}^{1} & = & \left(1-\beta_{n}^{1}\right) f_{n}^{2}+\beta_{n}^{1} u_{n}^{1}, \\
f_{n}^{2} & = & \left(1-\beta_{n}^{2}\right) f_{n}+\beta_{n}^{2} u_{n}^{2}, \quad n=0,1,2, \ldots
\end{array}\right.
$$

where $u_{n+1} \in P_{\rho}^{T}\left(f_{n+1}\right), u_{n}^{1} \in P_{\rho}^{T}\left(f_{n}^{1}\right), u_{n}^{2} \in P_{\rho}^{T}\left(f_{n}^{2}\right)$, and the sequences $\left\{\alpha_{n}\right\}_{n=0}^{\infty},\left\{\beta_{n}^{1}\right\}_{n=0}^{\infty}$, and $\left\{\beta_{n}^{2}\right\}_{n=0}^{\infty}$ are in $[0,1)$ such that $\sum_{n=0}^{\infty} \alpha_{n}=\infty$.

The implicit Ishikawa iterative sequence $\left\{f_{n}\right\}_{n=0}^{\infty} \subset D$ is defined by:

$$
\left\{\begin{array}{ccc}
f_{0} & \in & D \\
f_{n+1} & = & \left(1-\alpha_{n}\right) f_{n}^{1}+\alpha_{n} u_{n+1}, \\
f_{n}^{1} & = & \left(1-\beta_{n}^{1}\right) f_{n}+\beta_{n}^{1} u_{n}^{1}, \quad n=0,1,2, \ldots
\end{array}\right.
$$

where $u_{n+1} \in P_{\rho}^{T}\left(f_{n+1}\right), u_{n}^{1} \in P_{\rho}^{T}\left(f_{n}^{1}\right),\left\{\alpha_{n}\right\}_{n=0}^{\infty} \subset[0,1),\left\{\beta_{n}^{1}\right\}_{n=0}^{\infty} \subset[0,1)$ and $\sum_{n=0}^{\infty} \alpha_{n}=\infty$. 
The implicit Mann iterative sequence $\left\{f_{n}\right\}_{n=0}^{\infty} \subset D$ is defined by:

$$
\left\{\begin{array}{ccc}
f_{0} & \in & D \\
f_{n+1} & = & \left(1-\alpha_{n}\right) f_{n}+\alpha_{n} u_{n+1}, n=0,1,2, \ldots
\end{array}\right.
$$

Multistep-type construction of fixed point...

where $u_{n+1} \in P_{\rho}^{T}\left(f_{n+1}\right),\left\{\alpha_{n}\right\}_{n=0}^{\infty} \subset[0,1)$ and $\sum_{n=0}^{\infty} \alpha_{n}=\infty$.

The following Lemmas will be needed in proving the main results.

Lemma 1.1. ([3]). Let $T: D \rightarrow P_{\rho}(D)$ be a multivalued mapping and $P_{\rho}^{T}(f)=\{g \in T f$ : $\left.\rho(f-g)=\operatorname{dist}_{\rho}(f, T f)\right\}$. Then the following are equivalent:

(1) $f \in F_{\rho}(T)$, that is, $f \in T f$.

(2) $P_{\rho}^{T}(f)=\{f\}$.

(3) $f \in F\left(P_{\rho}^{T}(f)\right)$, that is, $f \in P_{\rho}^{T}(f)$. Further $F_{\rho}(T)=F\left(P_{\rho}^{T}(f)\right)$ where $F\left(P_{\rho}^{T}(f)\right)$ represent the set of fixed points of $P_{\rho}^{T}(f)$.

Lemma 1.2. (see [13]). Let $\delta$ be a real number satisfying $0 \leq \delta<1$ and $\left\{\varepsilon_{n}\right\}_{n=0}^{\infty}$ and $\left\{\tau_{n}\right\}_{n=0}^{\infty}$ two sequences of positive or zero numbers, less than 1 , such that $\lim _{n \rightarrow \infty} \varepsilon_{n}=0$ and $\sum_{n=0}^{\infty} \tau_{n}=\infty$. Then any sequence of positive numbers $\left\{u_{n}\right\}_{n=0}^{\infty}$ satisfying any of the following properties converges to 0 :

(1) $u_{n+1} \leq \delta u_{n}+\varepsilon_{n}$ for all $n=0,1,2, \ldots$

(2) $u_{n+1} \leq\left(1-\tau_{n}\right) u_{n}$ for all $n=0,1,2, \ldots$

(3) $u_{n+1} \leq \varepsilon_{n}+\left(1-\tau_{n}\right) u_{n}$ for all $n=0,1,2, \ldots$ if in addition, $\left\{\tau_{n}\right\}_{n=0}^{\infty}$ is bounded away from 0 .

\section{Convergence results}

\subsection{Strong convergence results for explicit multistep iterative sequences in modular function} spaces

Theorem 2.1. Let $D$ be a $\rho$-closed, $\rho$-bounded and convex subset of a $\rho$-complete modular space $L_{\rho}$, and $T: D \rightarrow P_{\rho}(D)$ be a multivalued mapping such that $P_{\rho}^{T}$ is a $\rho$-quasicontractive-like mapping, satisfying contractive-like condition (1.8). Suppose that $F_{\rho}(T) \neq \varnothing$. Let $f_{0} \in D$ and $\left\{f_{n}\right\} \subset D$ be defined by the explicit multistep iterative sequence (1.9), where the sequences $\left\{\alpha_{n}\right\}_{n=0}^{\infty},\left\{\beta_{n}^{i}\right\}_{n=0}^{\infty} \subset[0,1),(i=1,2, \ldots, k-1)$ are such that $\sum_{0}^{\infty} \alpha_{n}=\infty$. Then the explicit multistep iterative sequence (1.9) converges strongly to the fixed point of $T$.

Proof. Let $f \in F_{\rho}(T)$; from Lemma 1.1, $P_{\rho}^{T}(f)=\{f\}$ and $F_{\rho}(T)=F\left(P_{\rho}^{T}(f)\right)$.

Using the explicit multistep iterative sequence (1.9) and the convexity of $\rho$, we obtain the following estimate:

$$
\begin{gathered}
\rho\left(f_{n+1}-f\right)=\rho\left[\left(1-\alpha_{n}\right) f_{n}+\alpha_{n} v_{n}^{1}-f\right] \\
\quad=\rho\left[\left(1-\alpha_{n}\right)\left(f_{n}-f\right)+\alpha_{n}\left(v_{n}^{1}-f\right)\right] \\
\leq\left(1-\alpha_{n}\right) \rho\left(f_{n}-f\right)+\alpha_{n} \rho\left(v_{n}^{1}-f\right) .
\end{gathered}
$$

$v_{n}^{1} \in P_{\rho}^{T}\left(g_{n}^{1}\right)$ and $P_{\rho}^{T}(f)=\{f\}$ imply that: 


$$
\rho\left(v_{n}^{1}-f\right)=\operatorname{dist}_{\rho}\left(v_{n}^{1}, P_{\rho}^{T}(f)\right) \leq H_{\rho}\left(P_{\rho}^{T}\left(g_{n}^{1}\right), P_{\rho}^{T}(f)\right),
$$

which combined with (2.1) yields:

$$
\rho\left(f_{n+1}-f\right) \leq\left(1-\alpha_{n}\right) \rho\left(f_{n}-f\right)+\alpha_{n} H_{\rho}\left(P_{\rho}^{T}\left(g_{n}^{1}\right), P_{\rho}^{T}(f)\right) .
$$

In (1.8), letting $g=g_{n}^{1}$ and noting that $P_{\rho}^{T}(f)=\{f\}$ and $\varphi(0)=0$, we have:

$$
\begin{aligned}
H_{\rho}\left(P_{\rho}^{T}\left(g_{n}^{1}\right), P_{\rho}^{T}(f)\right) \leq & \delta \rho\left(g_{n}^{1}-f\right)+\varphi(f-h) \quad \forall h \in P_{\rho}^{T}(f) \\
\leq & \delta \rho\left(g_{n}^{1}-f\right) .
\end{aligned}
$$

Substituting (2.3) in (2.2), we obtain

$$
\rho\left(f_{n+1}-f\right) \leq\left(1-\alpha_{n}\right) \rho\left(f_{n}-f\right)+\delta \alpha_{n} \rho\left(g_{n}^{1}-f\right) .
$$

Similarly, from (1.9) and the convexity of $\rho$,

$$
\begin{aligned}
& \rho\left(g_{n}^{1}-f\right)=\rho\left[\left(1-\beta_{n}^{1}\right) f_{n}+\beta_{n}^{1} v_{n}^{2}-f\right] \\
& \quad=\rho\left[\left(1-\beta_{n}^{1}\right)\left(f_{n}-f\right)+\beta_{n}^{1}\left(v_{n}^{2}-f\right)\right] \\
& \quad \leq\left(1-\beta_{n}^{1}\right) \rho\left(f_{n}-f\right)+\beta_{n}^{1} \rho\left(v_{n}^{2}-f\right) .
\end{aligned}
$$

$v_{n}^{2} \in P_{\rho}^{T}\left(g_{n}^{2}\right)$ and $P_{\rho}^{T}(f)=\{f\}$ imply that:

$$
\rho\left(v_{n}^{2}-f\right)=\operatorname{dist}_{\rho}\left(v_{n}^{2}, P_{\rho}^{T}(f)\right) \leq H_{\rho}\left(P_{\rho}^{T}\left(g_{n}^{2}\right), P_{\rho}^{T}(f)\right),
$$

which combined with (2.5) yields:

$$
\rho\left(g_{n}^{1}-f\right) \leq\left(1-\beta_{n}^{1}\right) \rho\left(f_{n}-f\right)+\beta_{n}^{1} H_{\rho}\left(P_{\rho}^{T}\left(g_{n}^{2}\right), P_{\rho}^{T}(f)\right) .
$$

In (1.8), letting $g=g_{n}^{2}$ and noting that $P_{\rho}^{T}(f)=\{f\}$ and $\varphi(0)=0$, we get:

$$
\rho\left(g_{n}^{1}-f\right) \leq\left(1-\beta_{n}^{1}\right) \rho\left(f_{n}-f\right)+\delta \beta_{n}^{1} \rho\left(g_{n}^{2}-f\right) .
$$

Similarly, an application of (1.8) and (1.9) gives

$$
\rho\left(g_{n}^{2}-f\right) \leq\left(1-\beta_{n}^{2}\right) \rho\left(f_{n}-f\right)+\delta \beta_{n}^{2} \rho\left(g_{n}^{3}-f\right) .
$$

Also, an application of (1.8) and (1.9) gives

$$
\rho\left(g_{n}^{3}-f\right) \leq\left(1-\beta_{n}^{3}\right) \rho\left(f_{n}-f\right)+\delta \beta_{n}^{3} \rho\left(g_{n}^{4}-f\right) .
$$

Substituting (2.9) in (2.8), (2.8) in (2.7) and (2.7) in (2.4), and simplifying, we obtain

$$
\begin{gathered}
\rho\left(f_{n+1}-f\right) \leq\left[1-(1-\delta) \alpha_{n}-(1-\delta) \delta \alpha_{n} \beta_{n}^{1}-(1-\delta) \delta^{2} \alpha_{n} \beta_{n}^{1} \beta_{n}^{2}\right. \\
\left.-(1-\delta) \delta^{3} \alpha_{n} \beta_{n}^{1} \beta_{n}^{2} \beta_{n}^{3}\right]+\delta^{3} \alpha_{n} \beta_{n}^{1} \beta_{n}^{2} \beta_{n}^{3} \rho\left(g_{n}^{4}-f\right) .
\end{gathered}
$$

Continuing this process, an application of (1.8) and (1.9) gives

$$
\rho\left(g_{n}^{k-2}-f\right) \leq\left(1-\beta_{n}^{k-2}\right) \rho\left(f_{n}-f\right)+\delta \beta_{n}^{k-2} \rho\left(g_{n}^{k-1}-f\right) .
$$


and

$$
\rho\left(g_{n}^{k-1}-f\right) \leq\left(1-\beta_{n}^{k-1}\right) \rho\left(f_{n}-f\right)+\delta \beta_{n}^{k-1} \rho\left(f_{n}-f\right) .
$$

Substituting (2.12) and (2.11) in (2.10) inductively and simplifying, we obtain

$$
\begin{aligned}
\rho\left(f_{n+1}-f\right) \leq & {\left[1-(1-\delta) \alpha_{n}-\sum_{i=1}^{k-1}(1-\delta) \delta^{i} \alpha_{n} \beta_{n}^{1} \beta_{n}^{2} \ldots \beta_{n}^{i}\right.} \\
+ & \left.\delta^{k} \alpha_{n} \beta_{n}^{1} \beta_{n}^{2} \beta_{n}^{3} \beta_{n}^{4} \ldots \beta_{n}^{k-1}\right] \rho\left(f_{n}-f\right) \\
& \leq\left[1-(1-\delta) \alpha_{n}\right] \rho\left(f_{n}-f\right) .
\end{aligned}
$$

From (2.13), we inductively obtain

$$
\rho\left(f_{n+1}-f\right) \leq\left[\prod_{m=0}^{n}\left(1-(1-\delta) \alpha_{m}\right)\right] \rho\left(f_{0}-f\right) .
$$

Using that fact that $\delta \in[0,1)\left\{\alpha_{n}\right\}_{n=0}^{\infty} \subset[0,1)$ satisfying $\sum_{n=0}^{\infty} \alpha_{n}=\infty$, then from (2.14), we obtain

$$
\lim _{n \rightarrow \infty} \rho\left(f_{n+1}-f\right) \leq \lim _{n \rightarrow \infty} \prod_{m=0}^{n}\left[1-(1-\delta) \alpha_{m}\right] \rho\left(f_{0}-f\right)=0 .
$$

Therefore, $\left\{f_{n}\right\} \rho$-converges to $f \in F_{\rho}(T)$. The proof is complete.

Since the explicit Noor (1.10), explicit Ishikawa (1.11), explicit Mann (1.12) iterative sequences are special cases of the explicit multistep iterative sequence (1.9) (see [22] for details), then Theorem 2.1 leads to the following corollary:

Corollary 2.1. Let $D$ be a $\rho$-closed, $\rho$-bounded and convex subset of a $\rho$-complete modular space $L_{\rho}$, and $T: D \rightarrow P_{\rho}(D)$ be a multivalued mapping such that $P_{\rho}^{T}$ is a $\rho$ - quasicontractive-like mapping, satisfying contractive-like condition (1.8). Suppose that $F_{\rho}(T) \neq \varnothing /$. Let $f_{0} \in D$ and $\left\{f_{n}\right\} \subset D$ be defined by the explicit Noor (1.10), the explicit Ishikawa (1.11) and the explicit Mann (1.12) iterative sequences respectively, where the sequences $\left\{\alpha_{n}\right\}_{n=0}^{\infty},\left\{\beta_{n}^{1}\right\}_{n=0}^{\infty},\left\{\beta_{n}^{2}\right\}_{n=0}^{\infty} \subset[0,1)$ are such that $\sum_{0}^{\infty} \alpha_{n}=\infty$. Then:

(1) the explicit Noor iterative sequence (1.10) converges strongly to the fixed point of $T$.

(2) the explicit Ishikawa iterative sequence (1.11) converges strongly to the fixed point of $T$.

(3) the explicit Mann iterative sequence (1.12) converges strongly to the fixed point of $T$.

\subsection{Strong convergence results for explicit multistep-SP iterative sequences in modular function spaces}

Theorem 2.2. Let $D$ be a $\rho$-closed, $\rho$-bounded and convex subset of a $\rho$-complete modular space $L_{\rho}$, and $T: D \rightarrow P_{\rho}(D)$ be a multivalued mapping such that $P_{\rho}^{T}$ is a $\rho$ - quasicontractive-like mapping, satisfying contractive-like condition (1.8). Suppose that $F_{\rho}(T) \neq \emptyset /$. Let $f_{0} \in D$ and $\left\{f_{n}\right\} \subset D$ be defined by the explicit multistep-SP iterative sequence (1.13), where the sequences $\left\{\alpha_{n}\right\}_{n=0}^{\infty},\left\{\beta_{n}^{i}\right\}_{n=0}^{\infty} \subset[0,1),(i=1,2, \ldots, k-1)$ are 
AJMS

27,2

198

such that $\sum_{0}^{\infty} \alpha_{n}=\infty$. Then the explicit multistep-SP iterative sequence (1.13) $\rho$-converges to a fixed point of $T$.

Proof. Let $f \in F_{\rho}(T)$. From Lemma 1.1, we have that $P_{\rho}^{T}(f)=\{f\}$ and $F_{\rho}(T)=F\left(P_{\rho}^{T}(f)\right)$. Using the explicit multistep-SP iterative sequence (1.13) and the convexity of $\rho$, we obtain the following estimate:

$$
\begin{gathered}
\rho\left(f_{n+1}-f\right)=\rho\left[\left(1-\alpha_{n}\right) g_{n}^{1}+\alpha_{n} v_{n}^{1}-f\right] \\
\quad=\rho\left[\left(1-\alpha_{n}\right)\left(g_{n}^{1}-f\right)+\alpha_{n}\left(v_{n}^{1}-f\right)\right] \\
\leq\left(1-\alpha_{n}\right) \rho\left(g_{n}^{1}-f\right)+\alpha_{n} \rho\left(v_{n}^{1}-f\right) .
\end{gathered}
$$

Since $v_{n}^{1} \in P_{\rho}^{T}\left(g_{n}^{1}\right)$ and $P_{\rho}^{T}(f)=\{f\}$, we have

$$
\rho\left(v_{n}^{1}-f\right)=\operatorname{dist}_{\rho}\left(v_{n}^{1}, P_{\rho}^{T}(f)\right) \leq H_{\rho}\left(P_{\rho}^{T}\left(g_{n}^{1}\right), P_{\rho}^{T}(f)\right),
$$

which combined with (2.16) yields:

$$
\rho\left(f_{n+1}-f\right) \leq\left(1-\alpha_{n}\right) \rho\left(g_{n}^{1}-f\right)+\alpha_{n} H_{\rho}\left(P_{\rho}^{T}\left(g_{n}^{1}\right), P_{\rho}^{T}(f)\right) .
$$

In (1.8), letting $g=g_{n}^{1}$ and noting that $P_{\rho}^{T}(f)=\{f\}$ and $\varphi(0)=0$, we get

$$
H_{\rho}\left(P_{\rho}^{T}\left(g_{n}^{1}\right), P_{\rho}^{T}(f)\right) \leq \delta \rho\left(g_{n}^{1}-f\right)+\varphi(0)=\delta \rho\left(g_{n}^{1}-f\right) .
$$

Substituting (2.18) in (2.17), we obtain

$$
\begin{gathered}
\rho\left(f_{n+1}-f\right) \leq\left(1-\alpha_{n}\right) \rho\left(g_{n}^{1}-f\right)+\delta \alpha_{n} \rho\left(g_{n}^{1}-f\right) \\
=\left[1-(1-\delta) \alpha_{n}\right] \rho\left(g_{n}^{1}-f\right) .
\end{gathered}
$$

Next, from (1.13) and the convexity of $\rho$,

$$
\begin{gathered}
\rho\left(g_{n}^{1}-f\right)=\rho\left[\left(1-\beta_{n}^{1}\right) g_{n}^{2}+\beta_{n}^{1} v_{n}^{2}-f\right] \\
=\rho\left[\left(1-\beta_{n}^{1}\right)\left(g_{n}^{2}-f\right)+\beta_{n}^{1}\left(v_{n}^{2}-f\right)\right] \\
\leq\left(1-\beta_{n}^{1}\right) \rho\left(g_{n}^{2}-f\right)+\beta_{n}^{1} \rho\left(v_{n}^{2}-f\right) .
\end{gathered}
$$

Since $v_{n}^{2} \in P_{\rho}^{T}\left(g_{n}^{2}\right)$ and $P_{\rho}^{T}(f)=\{f\}$, we have

$$
\rho\left(v_{n}^{2}-f\right)=\operatorname{dist}_{\rho}\left(v_{n}^{2}, P_{\rho}^{T}(f)\right) \leq H_{\rho}\left(P_{\rho}^{T}\left(g_{n}^{2}\right), P_{\rho}^{T}(f)\right),
$$

which combined with (2.20) yields:

$$
\rho\left(g_{n}^{1}-f\right) \leq\left(1-\beta_{n}^{1}\right) \rho\left(g_{n}^{2}-f\right)+\beta_{n}^{1} H_{\rho}\left(P_{\rho}^{T}\left(g_{n}^{2}\right), P_{\rho}^{T}(f)\right) .
$$

Using (1.8) with $g=g_{n}^{2}$ in (2.21) and noting that $\varphi(0)=0$ and $P_{\rho}^{T}(f)=\{f\}$, then we get the following:

$$
\begin{gathered}
\rho\left(g_{n}^{1}-f\right) \leq\left(1-\beta_{n}^{1}\right) \rho\left(g_{n}^{2}-f\right)+\delta \beta_{n}^{1} \rho\left(g_{n}^{2}-f\right) \\
=\left[1-(1-\delta) \beta_{n}^{1}\right] \rho\left(g_{n}^{2}-f\right) .
\end{gathered}
$$

Similarly, an application of (1.8) and (1.13) gives

$$
\rho\left(g_{n}^{2}-f\right) \leq\left(1-\beta_{n}^{2}\right) \rho\left(g_{n}^{3}-f\right)+\delta \beta_{n}^{2} \rho\left(g_{n}^{3}-f\right)
$$




$$
=\left[1-(1-\delta) \beta_{n}^{2}\right] \rho\left(g_{n}^{3}-f\right) .
$$

Multistep-type

Also, an application of (1.8) and (1.13) gives

$$
\begin{aligned}
\rho\left(g_{n}^{3}-f\right) & \leq\left(1-\beta_{n}^{3}\right) \rho\left(g_{n}^{4}-f\right)+\delta \beta_{n}^{3} \rho\left(g_{n}^{4}-f\right) \\
= & {\left[1-(1-\delta) \beta_{n}^{3}\right] \rho\left(g_{n}^{4}-f\right) . }
\end{aligned}
$$

Continuing this process, an application of (1.8) and (1.13) gives

and

$$
\begin{aligned}
\rho\left(g_{n}^{k-2}-f\right) \leq & \left(1-\beta_{n}^{k-2}\right) \rho\left(g_{n}^{k-1}-f\right)+\delta \beta_{n}^{k-2} \rho\left(g_{n}^{k-1}-f\right) \\
= & {\left[1-(1-\delta) \beta_{n}^{k-2}\right] \rho\left(g_{n}^{k-1}-f\right) . } \\
\rho\left(g_{n}^{k-1}-f\right) & \leq\left(1-\beta_{n}^{k-1}\right) \rho\left(f_{n}-f\right)+\delta \beta_{n}^{k-1} \rho\left(f_{n}-f\right) \\
& =\left[1-(1-\delta) \beta_{n}^{k-1}\right] \rho\left(f_{n}-f\right) .
\end{aligned}
$$

Substituting (2.22)-(2.26) in (2.19) inductively and simplifying, we obtain

$$
\begin{gathered}
\rho\left(f_{n+1}-f\right) \leq\left(\left[1-(1-\delta) \alpha_{n}\right] \prod_{i=1}^{k 1}\left[1-(1-\delta) \beta_{n}^{i}\right]\right) \rho\left(f_{n}-f\right) \\
\leq\left[1-(1-\delta) \alpha_{n}\right] \rho\left(f_{n}-f\right) .
\end{gathered}
$$

From (2.27), we inductively obtain

$$
\rho\left(f_{n+1}-f\right) \leq \prod_{m=0}^{n}\left[1-(1-\delta) \alpha_{m}\right] \rho\left(f_{0}-f\right) .
$$

Using that fact that $\delta \in[0,1)\left\{\alpha_{n}\right\}_{n=0}^{\infty} \subset[0,1)$ satisfying $\sum_{n=0}^{\infty} \alpha_{n}=\infty$, then from (2.28), we obtain

$$
\lim _{n \rightarrow \infty} \rho\left(f_{n+1}-f\right) \leq \lim _{n \rightarrow \infty} \prod_{m=0}^{n}\left[1-(1-\delta) \alpha_{m}\right] \rho\left(f_{0}-f\right)=0 .
$$

Therefore, $\lim _{n \rightarrow \infty} \rho\left(f_{n}-f\right)=0$, where $f \in F_{\rho}(T)$. The proof is complete.

Theorem 2.2 leads to the following corollary:

Corollary 2.2. Let $D$ be a $\rho$-closed, $\rho$-bounded and convex subset of a $\rho$-complete modular space $L_{\rho}$, and $T: D \rightarrow P_{\rho}(D)$ be a multivalued mapping such that $P_{\rho}^{T}$ is a $\rho$-quasicontractive-like mapping, satisfying contractive-like condition (1.8). Suppose that $F_{\rho}(T) \neq \varnothing$. Let $f_{0} \in D$ and $\left\{f_{n}\right\} \subset D$ be defined by the explicit SP iterative sequence (1.14), with the sequences $\left\{\alpha_{n}\right\}_{n=0}^{\infty},\left\{\beta_{n}^{1}\right\}_{n=0}^{\infty},\left\{\beta_{n}^{2}\right\}_{n=0}^{\infty} \subset[0,1)$ such that $\sum_{0}^{\infty} \alpha_{n}=\infty$. Then, the explicit SP iterative sequence (1.14) $\rho$-converges strongly to a fixed point of $T$.

\subsection{Strong convergence results for implicit multistep iterative sequences in modular function spaces}

Theorem 2.3. Let $D$ be a $\rho$-closed, $\rho$-bounded and convex subset of a $\rho$-complete modular space $L_{\rho}$. Let $T: D \rightarrow P_{\rho}(D)$ be a multivalued mapping satisfying property (I) and such that $P_{\rho}^{T}$ is a $\rho$-quasi-contractive-like mapping, satisfying contractivelike condition (1.8). Suppose that $F_{\rho}(T) \neq \emptyset /$. Let $f_{0} \in D$ and $\left\{f_{n}\right\} \subset D$ be defined by the implicit multistep iterative sequence (1.15), where the sequences $\left\{\alpha_{n}\right\}_{n=0}^{\infty},\left\{\beta_{n}^{i}\right\}_{n=0}^{\infty} \subset[0,1)$ 
AJMS

27,2

200

$(i=1,2, \ldots, k-1)$ are such that $\sum_{0}^{\infty} \alpha_{n}=\infty$. Then, the implicit multistep iterative sequence (1.15) $\rho$-converges strongly to a fixed point of $T$.

Proof. Let $f \in F_{\rho}(T)$. From Lemma 1.1, we have that $P_{\rho}^{T}(f)=\{f\}$ and $F_{\rho}(T)=F\left(P_{\rho}^{T}(f)\right)$.

Using implicit multistep iterative sequence (1.15) and the convexity of $\rho$, we obtain the following estimate:

$$
\begin{gathered}
\rho\left(f_{n+1}-f\right)=\rho\left[\left(1-\alpha_{n}\right) f_{n}^{1}+\alpha_{n} u_{n+1}-f\right] \\
=\rho\left[\left(1-\alpha_{n}\right)\left(f_{n}^{1}-f\right)+\alpha_{n}\left(u_{n+1}-f\right)\right] \\
\leq\left(1-\alpha_{n}\right) \rho\left(f_{n}^{1}-f\right)+\alpha_{n} \rho\left(u_{n+1}-f\right)
\end{gathered}
$$

Since $u_{n+1} \in P_{\rho}^{T}\left(f_{n+1}\right)$ and $P_{\rho}^{T}(f)=\{f\}$,

$$
\rho\left(u_{n+1}-f\right) \leq \operatorname{dist}_{\rho}\left(u_{n+1}, P_{\rho}^{T}(f)\right) \leq H_{\rho}\left(P_{\rho}^{T}\left(f_{n+1}\right), P_{\rho}^{T}(f)\right),
$$

which combined with (2.30) gives

$$
\rho\left(f_{n+1}-f\right) \leq\left(1-\alpha_{n}\right) \rho\left(f_{n}^{1}-f\right)+\alpha_{n} H_{\rho}\left(P_{\rho}^{T}\left(f_{n+1}\right), P_{\rho}^{T}(f)\right) .
$$

In (1.8), by letting $g=f_{n+1}$ and noting that $\varphi(0)=0$ and $P_{\rho}^{T}(f)=\{f\}$, we get:

$$
H_{\rho}\left(P_{\rho}^{T}\left(f_{n+1}\right), P_{\rho}^{T}(f)\right) \leq \delta \rho\left(f_{n+1}-f\right)+\varphi_{\rho}(0)=\delta \rho\left(f_{n+1}-f\right) .
$$

Substituting (2.32) in (2.31), we obtain

$$
\rho\left(f_{n+1}-f\right) \leq\left(1-\alpha_{n}\right) \rho\left(f_{n}^{1}-f\right)+\delta \alpha_{n} \rho\left(f_{n+1}-f\right)
$$

That is,

$$
\rho\left(f_{n+1}-f\right) \leq\left[\frac{1-\alpha_{n}}{1-\delta \alpha_{n}}\right] \rho\left(f_{n}^{1}-f\right) .
$$

Next, from (1.15) and the convexity of $\rho$, we have

$$
\begin{gathered}
\rho\left(f_{n}^{1}-f\right)=\rho\left[\left(1-\beta_{n}^{1} f_{n}^{2}+\beta_{n}^{1}\right) u_{n}^{1}-f\right] \\
=\rho\left[\left(1-\beta_{n}^{1}\right)\left(f_{n}^{2}-f\right)+\beta_{n}^{1}\left(u_{n}^{1}-f\right)\right] \\
=\left(1-\beta_{n}^{1}\right) \rho\left(f_{n}^{2}-f\right)+\beta_{n}^{1} \rho\left(u_{n}^{1}-f\right) .
\end{gathered}
$$

Since $u_{n}^{1} \in P_{\rho}^{T}\left(f_{n}^{1}\right)$ and $P_{\rho}^{T}(f)=\{f\}$,

$$
\rho\left(u_{n}^{1}, f\right)=\operatorname{dist}_{\rho}\left(u_{n}^{1}, P_{\rho}^{T}(f)\right) \leq H_{\rho}\left(P_{\rho}^{T}\left(f_{n}^{1}\right), P_{\rho}^{T}(f)\right),
$$

which combined with (2.34) gives:

$$
\rho\left(f_{n}^{1}-f\right) \leq\left(1-\beta_{n}^{1}\right) \rho\left(f_{n}^{2}-f\right)+\beta_{n}^{1} H_{\rho}\left(P_{\rho}^{T}\left(f_{n}^{1}\right), P_{\rho}^{T}(f)\right) .
$$

By letting $g=f_{n}^{1}$ in (1.8) and noting that $\varphi(0)=0$ and $P_{\rho}^{T}(f)=\{f\}$, we get:

$$
H_{\rho}\left(P_{\rho}^{T}\left(f_{n}^{1}\right), P_{\rho}^{T}(f)\right) \leq \delta \rho\left(f_{n}^{1}-f\right)+\varphi_{\rho}(0)=\delta \rho\left(f_{n}^{1}-f\right)
$$

Substituting (2.36) in (2.35), we obtain

$$
\rho\left(f_{n}^{1}-f\right) \leq\left(1-\beta_{n}^{1}\right) \rho\left(f_{n}^{2}-f\right)+\delta \beta_{n}^{1} \rho\left(f_{n}^{1}-f\right)
$$


That is,

$$
\rho\left(f_{n}^{1}-f\right) \leq\left[\frac{1-\beta_{n}^{1}}{1-\delta \beta_{n}^{1}}\right] \rho\left(f_{n}^{2}-f\right) .
$$

Similarly, an application of (1.8) and (1.15) gives

$$
\begin{gathered}
\rho\left(f_{n}^{2}-f\right) \leq\left[\frac{1-\beta_{n}^{2}}{1-\delta \beta_{n}^{2}}\right] \rho\left(f_{n}^{3}-f\right) . \\
\rho\left(f_{n}^{3}-f\right) \leq\left[\frac{1-\beta_{n}^{3}}{1-\delta \beta_{n}^{3}}\right] \rho\left(f_{n}^{4}-f\right) . \\
\vdots \\
\rho\left(f_{n}^{k-2}-f\right) \leq\left[\frac{1-\beta_{n}^{k-2}}{1-\delta \beta_{n}^{k-2}}\right] \rho\left(f_{n}^{k-1}-f\right) . \\
\rho\left(f_{n}^{k-1}-f\right) \leq\left[\frac{1-\beta_{n}^{k-1}}{1-\delta \beta_{n}^{k-1}}\right] \rho\left(f_{n}-f\right) .
\end{gathered}
$$

Substituting (2.37)-(2.40) in (2.33) inductively and simplifying, we obtain

$$
\rho\left(f_{n+1}-f\right) \leq\left[\frac{1-\alpha_{n}}{1-\delta \alpha_{n}}\right]\left[\prod_{i=1}^{k 1} \frac{1-\beta_{n}^{i}}{1-\delta \beta_{n}^{i}}\right] \rho\left(f_{n}-f\right) .
$$

Observe that

$$
\frac{1-\alpha_{n}}{1-\delta \alpha_{n}} \leq 1-\alpha_{n}+\delta \alpha_{n},\left[\frac{1-\beta_{n}^{i}}{1-\delta \beta_{n}^{i}}\right] \leq 1-\beta_{n}^{i}+\delta \beta_{n}^{i}, i=1, \ldots, k-1
$$

Substituting (2.43) in (2.42) and simplifying, we obtain

$$
\rho\left(f_{n+1}-f\right) \leq\left[1-(1-\delta) \alpha_{n}\right] \rho\left(f_{n}-f\right) .
$$

From (2.44), we inductively obtain

$$
\rho\left(f_{n+1}-f\right) \leq \prod_{m=0}^{n}\left[1-(1-\delta) \alpha_{m}\right] \rho\left(f_{0}-f\right) .
$$

Using that fact that $\delta \in[0,1)\left\{\alpha_{n}\right\}_{n=0}^{\infty} \subset[0,1)$ satisfying $\sum_{n=0}^{\infty} \alpha_{n}=\infty$, then from (2.45), we obtain

$$
\lim _{n \rightarrow \infty} \rho\left(f_{n+1}-f\right) \leq \lim _{n \rightarrow \infty} \prod_{m=0}^{n}\left[1-(1-\delta) \alpha_{m}\right] \rho\left(f_{0}-f\right)=0 .
$$

Therefore, $\lim _{n \rightarrow \infty} \rho\left(f_{n}-f\right)=0$, with $f \in F_{\rho}(T)$. The proof is complete.

Theorem 2.3 leads to the following corollary:

Corollary 2.3. Let $D$ be a $\rho$-closed, $\rho$-bounded and convex subset of a $\rho$-complete modular space $L_{\rho}$. Let $T: D \rightarrow P_{\rho}(D)$ be a multivalued mapping satisfying property (I), such 
AJMS

27,2

202

that $P_{\rho}^{T}$ is a $\rho$-quasi-contractive-like mapping, satisfying contractive-like condition (1.8). Suppose that $F_{\rho}(T) \neq \varnothing$. Let $f_{0} \in D$ and $\left\{f_{n}\right\} \subset D$ be defined by the implicit Noor (1.16), implicit Ishikawa (1.17) and implicit Mann (1.18) iterative sequences respectively, where the sequences $\left\{\alpha_{n}\right\}_{n=0}^{\infty},\left\{\beta_{n}^{1}\right\}_{n=0}^{\infty},\left\{\beta_{n}^{2}\right\}_{n=0}^{\infty} \subset[0,1)$ are such that $\sum_{0}^{\infty} \alpha_{n}=\infty$. Then:

(1) the implicit Noor iterative sequence (1.16) converges strongly to the fixed point of $T$.

(2) the implicit Ishikawa iterative sequence (1.17) converges strongly to the fixed point of $T$.

(3) the implicit Mann iterative sequence (1.18) converges strongly to the fixed point of $T$.

\section{Stability results for strong $\rho$-quasi-contractive-like maps}

In this section, conditions for some stability types of the explicit and implicit multistep iterative sequences are stated and backed by proofs in the framework of modular function spaces.

The first important result on $T$ - stable single mappings was proved by Ostrowski [18] for Picard iteration. Berinde [13], presented useful explanation on how to obtain the stability of various iterative sequences. Okeke and Khan [7] gave a similar version of stability results for multivalued mapping in modular function spaces.

In this paper, we introduce two other versions of $\rho$-stability and attempt to relate them with the concept of $\rho$-stability in literature.

Definition 3.1. Let $D$ be a nonempty $\rho$-closed, $\rho$-bounded and convex subset of a $\rho$-complete modular space $L_{\rho}$, and $T: D \rightarrow P_{\rho}(D)$ be a multivalued mapping with $F_{\rho}(T) \neq \varnothing$. Suppose that a fixed-point iterative sequence defined by

$$
f_{n+1}=F\left(T, f_{n}\right)
$$

with initial guess $f_{0} \in D$ and $F$ is a given function, converges to a fixed point $f$ of $T$. Let $\left\{h_{n}\right\}_{n=0}^{\infty}$ be an arbitrary sequence in $D$. The fixed-point iterative sequence is said to be:

(1) $\rho$-stable with respect to $T$ if and only if

$$
\lim _{n \rightarrow \infty} \varepsilon_{n}=0 \Rightarrow \lim _{n \rightarrow \infty} h_{n}=f, \text { where } \varepsilon_{n}=\rho\left(h_{n+1}-F\left(T, h_{n}\right)\right) .
$$

(2) relatively $\rho$-stable with respect to $T$ if and only if

$$
\lim _{n \rightarrow \infty} \delta_{n}=0 \Rightarrow \lim _{n \rightarrow \infty} h_{n}=f, \text { where } \delta_{n}=\rho\left(h_{n+1}-f\right)-\rho\left(F\left(T, h_{n}\right)-f\right) .
$$

(3) weakly $\rho$-stable with respect to $T$ if and only if

$$
\sup _{\lambda \in(0,1]} \lambda \rho\left(\frac{h_{n+1}-F\left(T, h_{n}\right)}{\lambda}\right) \rightarrow 0 \Rightarrow \inf _{\lambda \in[1, \infty)} \lambda \rho\left(\frac{h_{n}-f}{\lambda}\right) \rightarrow 0 .
$$

The term "relatively" in (2) is employed because the premise of the convergence of $\left\{h_{n}\right\}$ to $f$ is hinged to the fact that $\rho\left(h_{n+1}-f\right)$ and $\rho\left(F\left(T, h_{n}\right)-f\right)$ get closer to each other as $n$ increases. It is not known if this concept is directly related to $\rho$-stability as defined in [7]. If $\rho$ satisfies the triangular inequality (an unwanted condition in this paper), the relation between relatively $\rho$-stability and $\rho$-stability is as follows: (1) a relative $\rho$-stable fixed-point iteration is $\rho$-stable if 
$\delta_{n}>0$ for $n$ sufficiently big since $\left|\delta_{n}\right| \leq \varepsilon_{n}$; (2) a $\rho$-stable fixed-point iteration is relatively $\rho$-stable if for $n$ sufficiently big, $\delta_{n}<0$ and $\left|\delta_{n}\right| \leq \varepsilon_{n}$.

However, a $\rho$-stable fixed-point iteration is weakly $\rho$-stable, hence the term "weakly."

In this sequel, we also introduce the following concepts of strong quasi-contractions particular to modular function spaces and compatible in some sense to the newly introduced stability notions.

Definition 3.2. Let $H_{\rho}(\cdot, \cdot)$ be the $\rho$-Hausdorff distance on the family $C_{\rho}\left(L_{\rho}\right)$ of nonempty $\rho$-closed $\rho$-bounded subsets of $L_{\rho}$, that is,

$$
H_{\rho}(A, B)=\max \left\{\sup _{f \in A} \operatorname{dist}_{\rho}(f, B), \sup _{g \in B} \operatorname{dist}_{\rho}(g, A)\right\}, A, B \in C_{\rho}\left(L_{\rho}\right) .
$$

A multivalued map $T: D \rightarrow C_{\rho}\left(L_{\rho}\right)$ is said to be an:

(1) $m$-strong $\rho$-contraction mapping, where $m \in \mathbb{N}$, if there exists a constant $\delta \in[0,1)$ such that

$$
H_{\rho}(T f, T g) \leq m \delta \rho\left(\frac{f-g}{m}\right), \forall f, g \in D ;
$$

(If $\delta=1$ in (3.5), $T$ is said to be an $m$-strong $\rho$-nonexpansive mapping)

(2) $m$-strong $\rho$-quasi-contractive mapping, where $m \in \mathbb{N}$, if

$$
H_{\rho}(T f, T g) \leq m \delta \rho\left(\frac{f-g}{m}\right)+L \rho(h-f), \forall f, g \in D \quad \forall h \in T f, L \geq 0
$$

(If $\delta=1$ in (3.6), $T$ is said to be an $m$-strong $\rho$-quasi-contractive mapping)

(3) $m$-strong $\rho$-quasi-contractive-like mapping, where $m \in \mathbb{N}$, if

$$
H_{\rho}(T f, T g) \leq m \delta \rho\left(\frac{f-g}{m}\right)+\varphi(\rho(h-f)), \forall f, g \in D \forall h \in T f .
$$

where $\varphi: \mathbb{R}^{+} \rightarrow \mathbb{R}^{+}$is a monotone increasing function with $\varphi(0)=0$.(If $\delta=1 \mathrm{in}(3.7), T$ is said to be a $m$-strong $\rho$-quasi-contractive-like mapping).

Given any $m \in \mathbb{N}$, an $m$-strong $\rho$-contraction (resp. $\rho$-quasi-contractive mapping, or a $\rho$-quasi-contractive-like mapping) is a $\rho$-contraction (resp. $\rho$-quasi-contractive mapping, or a $\rho$-quasi-contractive-like mapping), thus, the convergence results in the previous section hold for $m$-strong $\rho$-quasi-contractive-like mappings. The converse is trivial when $m=1$.

\subsection{Stability results for explicit multistep iterative sequences in modular function spaces}

Theorem 3.1. Let $D$ be a $\rho$-closed, $\rho$-bounded and convex subset of a $\rho$-complete modular space $L_{\rho}$, and $T: D \rightarrow P_{\rho}(D)$ be a multivalued mapping such that $P_{\rho}^{T}$ is an $m$-strong $\rho$-quasi-contractive-like mapping, satisfying contractive-like condition (3.7), where $m \in \mathbb{N}$. Suppose that $F_{\rho}(T) \neq \varnothing$. Let $f_{0} \in D$ and $\left\{f_{n}\right\} \subset D$ be defined by the explicit multistep iterative sequence (1.9), where the sequences $\left\{\alpha_{n}\right\}_{n=0}^{\infty},\left\{\beta_{n}^{i}\right\}_{n=0}^{\infty} \subset[0,1)(i=1,2, \ldots, k-1)$ are such that $\left\{\alpha_{n}\right\}_{n=0}^{\infty}$ is bounded away from 0 . Then, (1.9) is:

(1) relatively $\rho$-stable with respect to $T$ if $m=1$;

(2) weakly $\rho$-stable with respect to $T$ if $m>1$.
Multistep-type construction of fixed point... 
AJMS

27,2

204

(3) $\rho$-stable with respect to $T$ if $m>1$ and $\forall g \in D \rho(g-f)=m \rho\left(\frac{g-f}{m}\right)$ where $f \in F_{\rho}(T)$ (in this case, $P_{\rho}^{T}$ is a $\rho$-quasi-contractive-like map).

Proof. Let $\left\{\alpha_{n}\right\}_{n=0}^{\infty},\left\{\beta_{n}^{i}\right\}_{n=0}^{\infty} \subset[0,1)(i=1,2, \ldots, k-1)$ be sequences such that $\left\{\alpha_{n}\right\}_{n=0}^{\infty}$ is bounded away from 0 .

Let $\left\{h_{n}\right\}_{n=0}^{\infty}$ be an arbitrary sequence in $D$ and set:

$$
\left\{\begin{array}{l}
\varepsilon_{n}=\rho\left(h_{n+1}-\left(1-\alpha_{n}\right) h_{n}-\alpha_{n} z_{n}^{1}\right) \\
\delta_{n}=\rho\left(h_{n+1}-f\right)-\rho\left(\left(1-\alpha_{n}\right) h_{n}+\alpha_{n} z_{n}^{1}-f\right) \\
\gamma_{n}=\sup _{\lambda \in(0,1]} \lambda \rho\left(\frac{h_{n+1}-\left(1-\alpha_{n}\right) h_{n}-\alpha_{n} z_{n}^{1}}{\lambda}\right) \\
s_{n}^{i}=\left(1-\beta_{n}^{i}\right) h_{n}+\beta_{n}^{i} z_{n}^{i+1}, \quad i=1,2, \ldots, k-2 \\
s_{n}^{k-1}=\left(1-\beta_{n}^{k-1}\right) h_{n}+\beta_{n}^{k-1} w_{n}, \quad n=0,1,2, \ldots
\end{array}\right.
$$

where $w_{n} \in P_{\rho}^{T}\left(h_{n}\right)$ and $z_{n}^{i} \in P_{\rho}^{T}\left(s_{n}^{i}\right), i=1,2, \ldots, k-1$.

Let:

$$
r_{n, m}=\left(1-\alpha_{n}\right) \rho\left(\frac{h_{n}-f}{m}\right)+\frac{\alpha_{n}}{m} \rho\left(z_{n}^{1}-f\right)
$$

By the convexity of $\rho$, we have:

$$
\begin{aligned}
\rho\left(h_{n+1}-f\right) & =\delta_{n}+\rho\left(\left(1-\alpha_{n}\right) h_{n}+\alpha_{n} z_{n}^{1}-f\right) \\
& =\delta_{n}+\rho\left(\left(1-\alpha_{n}\right)\left(h_{n}-f\right)+\alpha_{n}\left(z_{n}^{1}-f\right)\right) \\
& \leq \delta_{n}+r_{n, 1} .
\end{aligned}
$$

If $m>1$, we have:

$$
\begin{aligned}
\rho\left(\frac{h_{n+1}-f}{m}\right) & =\rho\left(\frac{\alpha_{n}}{m} \frac{h_{n+1}-\left(1-\alpha_{n}\right) h_{n}-\alpha_{n} z_{n}^{1}}{\alpha_{n}}+\left(1-\alpha_{n}\right) \frac{h_{n}-f}{m}+\frac{\alpha_{n}}{m}\left(z_{n}^{1}-f\right)\right) \\
& \leq \frac{\alpha_{n}}{m} \rho\left(\frac{h_{n+1}-\left(1-\alpha_{n}\right) h_{n}-\alpha_{n} z_{n}^{1}}{\alpha_{n}}\right)+r_{n, m} \\
& \leq \frac{\gamma_{n}}{m}+r_{n, m} .
\end{aligned}
$$

and if in addition $\forall g \in D \rho(g-f)=m \rho\left(\frac{g-f}{m}\right)$,

$$
\begin{aligned}
\rho\left(\frac{h_{n+1}-f}{m}\right) & =\rho\left(\frac{h_{n+1}-\left(1-\alpha_{n}\right) h_{n}-\alpha_{n} z_{n}^{1}}{m}+\frac{1-\alpha_{n}}{m}\left(h_{n}-f\right)+\frac{\alpha_{n}}{m}\left(z_{n}^{1}-f\right)\right) \\
& \leq \frac{1}{m} \varepsilon_{n}+r_{n, m} .
\end{aligned}
$$


Since $z_{n}^{1} \in P_{\rho}^{T}\left(s_{n}^{1}\right)$, then $\rho\left(z_{n}^{1}-f\right)=\operatorname{dist}_{\rho}\left(z_{n}^{1}, P_{\rho}^{T}(f)\right) \leq H_{\rho}\left(P_{\rho}^{T}\left(s_{n}^{1}\right), P_{\rho}^{T}(f)\right)$ hence:

$$
r_{n, m} \leq\left(1-\alpha_{n}\right) \rho\left(\frac{h_{n}-f}{m}\right)+\frac{\alpha_{n}}{m} H_{\rho}\left(P_{\rho}^{T}\left(s_{n}^{1}\right), P_{\rho}^{T}(f)\right) .
$$

Multistep-type construction of fixed point...

Using (3.7) and (3.8), and noting that $\varphi(0)=0$, we get the following:

$$
r_{n, m} \leq\left(1-\alpha_{n}\right) \rho\left(\frac{h_{n}-f}{m}\right)+\delta \alpha_{n} \rho\left(\frac{s_{n}^{1}-f}{m}\right)
$$

Using the convexity of $\rho$ in (3.8), and the fact that $z_{n}^{2} \in P_{\rho}^{T}\left(s_{n}^{2}\right)$, we have

$$
\begin{gathered}
\rho\left(\frac{s_{n}^{1}-f}{m}\right) \leq\left(1-\beta_{n}^{1}\right) \rho\left(\frac{h_{n}-f}{m}\right)+\beta_{n}^{1} \rho\left(\frac{z_{n}^{2}-f}{m}\right) \\
\leq\left(1-\beta_{n}^{1}\right) \rho\left(\frac{h_{n}-f}{m}\right)+\frac{\beta_{n}^{1}}{m} \operatorname{dist}_{\rho}\left(z_{n}^{2}, P_{\rho}^{T}(f)\right) \\
\leq\left(1-\beta_{n}^{1}\right) \rho\left(\frac{h_{n}-f}{m}\right)+\frac{\beta_{n}^{1}}{m} H_{\rho}\left(P_{\rho}^{T}\left(s_{n}^{2}\right), P_{\rho}^{T}(f)\right) .
\end{gathered}
$$

Using (3.7) and noting that $\varphi(0)=0$, then we get the following:

$$
\rho\left(\frac{s_{n}^{1}-f}{m}\right) \leq\left(1-\beta_{n}^{1}\right) \rho\left(\frac{h_{n}-f}{m}\right)+\delta \beta_{n}^{1} \rho\left(\frac{s_{n}^{2}-f}{m}\right) .
$$

Substituting (3.16) in (3.15), then in (3.14), we obtain

$$
\begin{gathered}
r_{n, m} \leq\left(1-\alpha_{n}\right) \rho\left(\frac{h_{n}-f}{m}\right)+\delta \alpha_{n} \rho\left(\frac{s_{n}^{1}-f}{m}\right) \\
\leq\left(1-\alpha_{n}\right) \rho\left(\frac{h_{n}-f}{m}\right)+\delta \alpha_{n}\left(1-\beta_{n}^{1}\right) \rho\left(\frac{h_{n}-f}{m}\right)+\delta^{2} \alpha_{n} \beta_{n}^{1} \rho\left(\frac{s_{n}^{2}-f}{m}\right) \\
\leq\left[1-(1-\delta) \alpha_{n}-\alpha_{n} \beta_{n}^{1} \delta\right] \rho\left(\frac{h_{n}-f}{m}\right)+\delta^{2} \alpha_{n} \beta_{n}^{1} \rho\left(\frac{s_{n}^{2}-f}{m}\right) .
\end{gathered}
$$

Similarly, successive applications of (1.8) and (3.3) give:

$$
\left\{\begin{array}{l}
\rho\left(\frac{s_{n}^{2}-f}{m}\right) \leq\left(1-\beta_{n}^{2}\right) \rho\left(\frac{h_{n}-f}{m}\right)+\delta \beta_{n}^{2} \rho\left(\frac{s_{n}^{3}-f}{m}\right) \\
\rho\left(\frac{s_{n}^{3}-f}{m}\right) \leq\left(1-\beta_{n}^{3}\right) \rho\left(\frac{h_{n}-f}{m}\right)+\delta \beta_{n}^{3} \rho\left(\frac{s_{n}^{4}-f}{m}\right) \\
\rho\left(\frac{s_{n}^{k-2}-f}{m}\right) \leq\left(1-\beta_{n}^{k-2}\right) \rho\left(\frac{h_{n}-f}{m}\right)+\delta \beta_{n}^{k-2} \rho\left(\frac{s_{n}^{k-1}-f}{m}\right) \\
\rho\left(\frac{s_{n}^{k-1}-f}{m}\right) \leq\left(1-\beta_{n}^{k-1}\right) \rho\left(\frac{h_{n}-f}{m}\right)+\delta \beta_{n}^{k-1} \rho\left(\frac{h_{n}-f}{m}\right)
\end{array}\right.
$$


AJMS

27,2

206

Substituting (3.18) in (3.17), and simplifying, we obtain

$$
r_{n, m} \leq\left[1-(1-\delta) \alpha_{n}\right] \rho\left(\frac{h_{n}-f}{m}\right) .
$$

Hence we have the equations:

$$
\rho\left(h_{n+1}-f\right) \leq \delta_{n}+\left[1-(1-\delta) \alpha_{n}\right] \rho\left(h_{n}-f\right)
$$

and if $m>1$,

$$
\rho\left(\frac{h_{n+1}-f}{m}\right) \leq \frac{\gamma_{n}}{m}+\left[1-(1-\delta) \alpha_{n}\right] \rho\left(\frac{h_{n}-f}{m}\right)
$$

and if in addition $\forall g \in D \rho(g-f)=m \rho\left(\frac{g-f}{m}\right)$,

$$
\rho\left(h_{n+1}-f\right) \leq \frac{1}{m} \varepsilon_{n}+\left[1-(1-\delta) \alpha_{n}\right] \rho\left(h_{n}-f\right) .
$$

(1) If $m=1$, then from (3.20) and Lemma 1.2, $\lim _{n \rightarrow \infty} \delta_{n}=0 \Rightarrow h_{n} \rightarrow f$. Thus, the fixedpoint iteration (1.9) is relatively $\rho$-stable.

(2) Suppose now that $m>1$ and that $\lim _{n \rightarrow \infty} \gamma_{n}=0$.

Then by (3.21) and Lemma 1.2, $\rho\left(\frac{h_{n}-f}{m}\right) \rightarrow 0$ and $m \rho\left(\frac{h_{n}-f}{m}\right) \rightarrow 0$. Thus, the fixed-point iteration (1.9) is weakly $\rho$-stable.

(3) Suppose that $m>1$ and that $\forall g \in D \rho(g-f)=m \rho\left(\frac{g-f}{m}\right)$. If $\lim _{n \rightarrow \infty} \varepsilon_{n}=0$, then by (3.22) and Lemma 1.2, $h_{n} \rightarrow f$. Thus, the fixed-point iteration (1.9) is $\rho$-stable.

Theorem 3.1 leads to the following corollary:

Corollary 3.1. Let $D$ be a $\rho$-closed, $\rho$-bounded and convex subset of a $\rho$-complete modular space $L_{\rho}$, and $T: D \rightarrow P_{\rho}(D)$ be a multivalued mapping such that $P_{\rho}^{T}$ is an $m$-strong $\rho$-quasi-contractive-like mapping, satisfying contractive-like condition (3.7), where $m \in \mathbb{N}$. Suppose that $F_{\rho}(T) \neq \emptyset /$. Let $f_{0} \in D$ and $\left\{f_{n}\right\} \subset D$ be the explicit Noor (1.10), the explicit Ishikawa (1.11) or the explicit Mann (1.12) iterative sequence, where the sequences $\left\{\alpha_{n}\right\}_{n=0}^{\infty},\left\{\beta_{n}^{1}\right\}_{n=0}^{\infty},\left\{\beta_{n}^{2}\right\}_{n=0}^{\infty} \subset[0,1)$ are such that $\left\{\alpha_{n}\right\}_{n=0}^{\infty}$ is bounded away from 0 . Then $\left\{f_{n}\right\}$ is

(1) relatively $\rho$-stable with respect to $T$ if $m=1$;

(2) weakly $\rho$-stable with respect to $T$ if $m>1$.

(3) $\rho$-stable with respect to $T$ if $m>1$ and $\forall g \in D \rho(g-f)=m \rho\left(\frac{g-f}{m}\right)$ where $f \in F_{\rho}(T)$ (in this case, $P_{\rho}^{T}$ is a $\rho$-quasi-contractive-like map).

\subsection{Stability results for explicit multistep-SP iterative sequences in modular function spaces}

Theorem 3.2. Let $D$ be a $\rho$-closed, $\rho$-bounded and convex subset of a $\rho$-complete modular space $L_{\rho}$, and $T: D \rightarrow P_{\rho}(D)$ be a multivalued mapping such that $P_{\rho}^{T}$ is an $m$-strong 
$\rho$-quasi-contractive-like mapping, satisfying contractive-like condition (3.7), where $m \in \mathbb{N}$. Suppose that $F_{\rho}(T) \neq \varnothing$. Let $f_{0} \in D$ and $\left\{f_{n}\right\} \subset D$ be defined by the explicit multistep iterative sequence (1.13), where the sequences $\left\{\alpha_{n}\right\}_{n=0}^{\infty},\left\{\beta_{n}^{i}\right\}_{n=0}^{\infty} \subset[0,1)(i=1,2, \ldots, k-1)$ are such that $\left\{\alpha_{n}\right\}_{n=0}^{\infty}$ is bounded away from 0 . Then, (1.13) is:

(1) relatively $\rho$-stable with respect to $T$ if $m=1$;

(2) weakly $\rho$-stable with respect to $T$ if $m>1$.

(3) $\rho$-stable with respect to $T$ if $m>1$ and $\forall g \in D \rho(g-f)=m \rho\left(\frac{g-f}{m}\right)$ where $f \in F_{\rho}(T)$ (in this case, $P_{\rho}^{T}$ is a $\rho$-quasi-contractive-like map).

Proof. The method of proof is similar to that of Theorem 3.1.

Theorem 3.2 leads to the following corollary:

Corollary 3.2. Let $D$ be a $\rho$-closed, $\rho$-bounded and convex subset of a $\rho$-complete modular space $L_{\rho}$, and $T: D \rightarrow P_{\rho}(D)$ be a multivalued mapping such that $P_{\rho}^{T}$ is an $m$-strong $\rho$-quasi-contractive-like mapping, satisfying contractive-like condition (3.7), where $m \in \mathbb{N}$. Suppose that $F_{\rho}(T) \neq \varnothing$. Let $f_{0} \in D$ and $\left\{f_{n}\right\} \subset D$ be defined by the explicit SP iterative sequence (1.14), with the sequences $\left\{\alpha_{n}\right\}_{n=0}^{\infty},\left\{\beta_{n}^{1}\right\}_{n=0}^{\infty},\left\{\beta_{n}^{2}\right\}_{n=0}^{\infty} \subset[0,1)$ such that $\left\{\alpha_{n}\right\}_{n=0}^{\infty}$ is bounded away from 0 . Then (1.14) is:

(1) relatively $\rho$-stable with respect to $T$ if $m=1$;

(2) weakly $\rho$-stable with respect to $T$ if $m>1$;

(3) $\rho$-stable with respect to $T$ if $m>1$ and $\forall g \in D \rho(g-f)=m \rho\left(\frac{g-f}{m}\right)$ where $f \in F_{\rho}(T)$ (in this case, $P_{\rho}^{T}$ is a $\rho$-quasi-contractive-like map).

\subsection{Stability results for implicit multistep iterative sequences in modular function spaces}

Theorem 3.3. Let $D$ be a $\rho$-closed, $\rho$-bounded and convex subset of a $\rho$-complete modular space $L_{\rho}$. Let $T: D \rightarrow P_{\rho}(D)$ be a multivalued mapping satisfying property (I), such that $P_{\rho}^{T}$ is an $m$-strong $\rho$-quasi-contractive-like mapping, satisfying contractivelike condition (3.7), where $m \in \mathbb{N}$. Suppose that $F_{\rho}(T) \neq \emptyset /$. Let $f_{0} \in D$ and $\left\{f_{n}\right\} \subset D$ be defined by the implicit multistep iterative sequence (1.15), where the sequences $\left\{\alpha_{n}\right\}_{n=0}^{\infty},\left\{\beta_{n}^{i}\right\}_{n=0}^{\infty} \subset[0,1)(i=1,2, \ldots, k-1)$ are such that $\left\{\alpha_{n}\right\}_{n=0}^{\infty}$ is bounded away from 0 . Then, (1.15) is:

(1) relatively $\rho$-stable with respect to $T$ if $m=1$;

(2) weakly $\rho$-stable with respect to $T$ if $m>1$.

(3) $\rho$-stable with respect to $T$ if $m>1$ and $\forall g \in D \rho(g-f)=m \rho\left(\frac{g-f}{m}\right)$ where $f \in F_{\rho}(T)$ (in this case, $P_{\rho}^{T}$ is a $\rho$-quasi-contractive-like map).

Proof.

Let $\left\{\alpha_{n}\right\}_{n=0}^{\infty},\left\{\beta_{n}^{i}\right\}_{n=0}^{\infty} \subset[0,1)$ be sequences such that $\left\{\alpha_{n}\right\}_{n=0}^{\infty}$ is bounded away from 0 . Suppose $f \in F_{\rho}(T)$. Let $\left\{h_{n}\right\}_{n=0}^{\infty}$ is an arbitrary sequence and set:
Multistep-type construction of fixed point... 


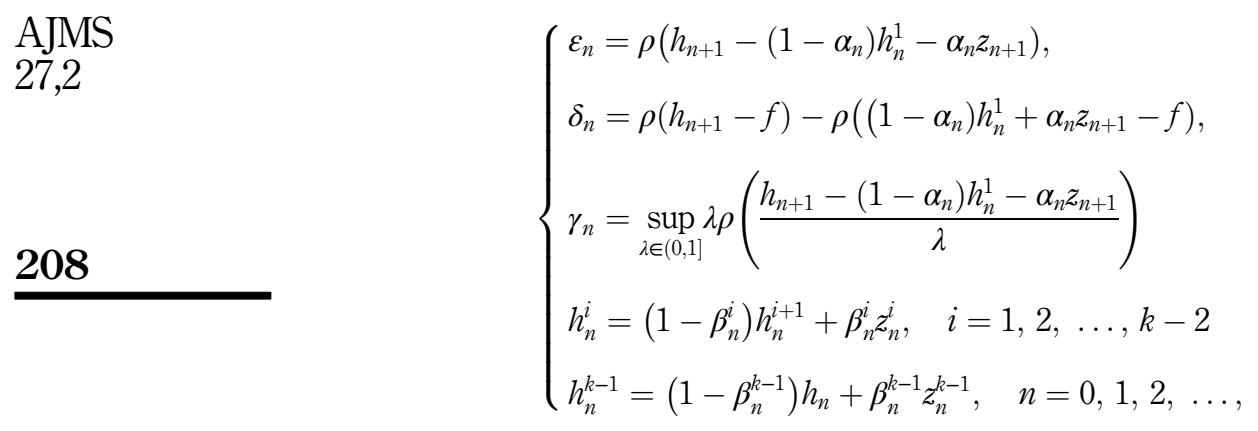

where $z_{n+1} \in P_{\rho}^{T}\left(h_{n+1}\right), z_{n}^{i} \in P_{\rho}^{T}\left(h_{n}^{i}\right), i=1,2, \ldots, k-1$.

Let:

$$
r_{n, m}=\left(1-\alpha_{n}\right) \rho\left(\frac{h_{n}^{1}-f}{m}\right)+\frac{\alpha_{n}}{m} \rho\left(z_{n+1}-f\right) .
$$

By the convexity of $\rho$, we have:

$$
\begin{aligned}
\rho\left(h_{n+1}-f\right) & =\delta_{n}+\rho\left(\left(1-\alpha_{n}\right) h_{n}^{1}+\alpha_{n} z_{n+1}-f\right) \\
& =\delta_{n}+\rho\left(\left(1-\alpha_{n}\right)\left(h_{n}^{1}-f\right)+\alpha_{n}\left(z_{n+1}-f\right)\right) \\
& \leq \delta_{n}+r_{n, 1} .
\end{aligned}
$$

If $m>1$, we have:

$$
\begin{aligned}
\rho\left(\frac{h_{n+1}-f}{m}\right) & =\rho\left(\frac{\alpha_{n}}{m} \frac{h_{n+1}-\left(1-\alpha_{n}\right) h_{n}^{1}-\alpha_{n} z_{n+1}}{\alpha_{n}}+\left(1-\alpha_{n}\right) \frac{h_{n}^{1}-f}{m}+\frac{\alpha_{n}}{m}\left(z_{n+1}-f\right)\right) \\
& \leq \frac{\alpha_{n}}{m} \rho\left(\frac{h_{n+1}-\left(1-\alpha_{n}\right) h_{n}^{1}-\alpha_{n} z_{n+1}}{\alpha_{n}}\right)+r_{n, m} \\
& \leq \frac{\gamma_{n}}{m}+r_{n, m} .
\end{aligned}
$$

and if in addition $\forall g \in D \rho(g-f)=m \rho\left(\frac{g-f}{m}\right)$,

$$
\begin{aligned}
\rho\left(\frac{h_{n+1}-f}{m}\right) & =\rho\left(\frac{h_{n+1}-\left(1-\alpha_{n}\right) h_{n}^{1}-\alpha_{n} z_{n+1}}{m}+\frac{1-\alpha_{n}}{m}\left(h_{n}^{1}-f\right)+\frac{\alpha_{n}}{m}\left(z_{n+1}-f\right)\right) \\
& \leq \frac{1}{m} \varepsilon_{n}+r_{n, m} .
\end{aligned}
$$


Since $z_{n+1} \in P_{\rho}^{T}\left(h_{n+1}\right)$, from (3.24) and (3.7) we have that:

$$
\begin{aligned}
r_{n, m} & =\left(1-\alpha_{n}\right) \rho\left(\frac{h_{n}^{1}-f}{m}\right)+\frac{\alpha_{n}}{m} \rho\left(z_{n+1}-f\right) \\
& =\left(1-\alpha_{n}\right) \rho\left(\frac{h_{n}^{1}-f}{m}\right)+\frac{\alpha_{n}}{m} \operatorname{dist}_{\rho}\left(z_{n+1}, P_{\rho}^{T}(f)\right) \\
& \leq\left(1-\alpha_{n}\right) \rho\left(\frac{h_{n}^{1}-f}{m}\right)+\frac{\alpha_{n}}{m} H_{\rho}\left(P_{\rho}^{T}\left(h_{n+1}\right), P_{\rho}^{T}(f)\right) \\
& \leq\left(1-\alpha_{n}\right) \rho\left(\frac{h_{n}^{1}-f}{m}\right)+\delta \alpha_{n} \rho\left(\frac{h_{n+1}-f}{m}\right) .
\end{aligned}
$$

Using the convexity of $\rho$ in (3.23), and the fact that $z_{n}^{1} \in P_{\rho}^{T}\left(h_{n}^{1}\right)$, we have

$$
\begin{aligned}
\rho\left(\frac{h_{n}^{1}-f}{m}\right) & \leq\left(1-\beta_{n}^{1}\right) \rho\left(\frac{h_{n}^{2}-f}{m}\right)+\beta_{n}^{1} \rho\left(\frac{z_{n}^{1}-f}{m}\right) \\
& \leq\left(1-\beta_{n}^{1}\right) \rho\left(\frac{h_{n}^{2}-f}{m}\right)+\frac{\beta_{n}^{1}}{m} \operatorname{dist}_{\rho}\left(z_{n}^{1}, P_{\rho}^{T}(f)\right) \\
& \leq\left(1-\beta_{n}^{1}\right) \rho\left(\frac{h_{n}^{2}-f}{m}\right)+\frac{\beta_{n}^{1}}{m} H_{\rho}\left(P_{\rho}^{T}\left(h_{n}^{1}\right), P_{\rho}^{T}(f)\right) \\
& \leq\left(1-\beta_{n}^{1}\right) \rho\left(\frac{h_{n}^{2}-f}{m}\right)+\delta \beta_{n}^{1} \rho\left(\frac{h_{n}^{1}-f}{m}\right) .
\end{aligned}
$$

Thus:

$$
\rho\left(\frac{h_{n}^{1}-f}{m}\right) \leq\left[\frac{1-\beta_{n}^{1}}{1-\delta \beta_{n}^{1}}\right] \rho\left(\frac{h_{n}^{2}-f}{m}\right) .
$$

Similarly, we have the following:

$$
\begin{gathered}
\rho\left(\frac{h_{n}^{2}-f}{m}\right) \leq\left[\frac{1-\beta_{n}^{2}}{1-\delta \beta_{n}^{2}}\right] \rho\left(\frac{h_{n}^{3}-f}{m}\right) \\
\vdots \\
\rho\left(\frac{h_{n}^{k-2}-f}{m}\right) \leq\left[\frac{1-\beta_{n}^{k-2}}{1-\delta \beta_{n}^{k-2}}\right] \rho\left(\frac{h_{n}^{k-1}-f}{m}\right)
\end{gathered}
$$


AJMS

27,2

$$
\rho\left(\frac{h_{n}^{k-1}-f}{m}\right) \leq\left[\frac{1-\beta_{n}^{k-1}}{1-\delta \beta_{n}^{k-1}}\right] \rho\left(\frac{h_{n}-f}{m}\right)
$$

Substituting (3.29) - (3.32), and simplifying, we obtain

\section{0}

$$
\begin{aligned}
r_{n, m} & \leq\left(1-\alpha_{n}\right)\left[\prod_{i=1}^{m} \frac{1-\beta_{n}^{i-1}}{1-\delta \beta_{n}^{i-1}}\right] \rho\left(\frac{h_{n}-f}{m}\right)+\delta \alpha_{n} \rho\left(\frac{h_{n+1}-f}{m}\right) \\
& \leq\left(1-\alpha_{n}\right) \rho\left(\frac{h_{n}-f}{m}\right)+\delta \alpha_{n} \rho\left(\frac{h_{n+1}-f}{m}\right) .
\end{aligned}
$$

Hence, substituting (3.33) in (3.25)-(3.27), we have the equations:

$$
\rho\left(h_{n+1}-f\right) \leq \delta_{n}+\left(\frac{1-\alpha_{n}}{1-\delta \alpha_{n}}\right) \rho\left(h_{n}-f\right)
$$

and if $m>1$,

$$
\rho\left(\frac{h_{n+1}-f}{m}\right) \leq \frac{\gamma_{n}}{m}+\left(\frac{1-\alpha_{n}}{1-\delta \alpha_{n}}\right) \rho\left(\frac{h_{n}-f}{m}\right) .
$$

and if in addition $\forall g \in D \rho(g-f)=m \rho\left(\frac{g-f}{m}\right)$,

$$
\rho\left(\frac{h_{n+1}-f}{m}\right) \leq \frac{\varepsilon_{n}}{m}+\left(\frac{1-\alpha_{n}}{1-\delta \alpha_{n}}\right) \rho\left(\frac{h_{n}-f}{m}\right) .
$$

(1) If $m=1$, then from (3.34) and Lemma 1.2, $\lim _{n \rightarrow \infty} \delta_{n}=0 \Rightarrow h_{n} \rightarrow f$. Thus the fixedpoint iteration (1.15) is relatively $\rho$-stable.

(2) Suppose now that $m>1$ and that $\lim _{n \rightarrow \infty} \gamma_{n}=0$.

Then by (3.35) and Lemma 1.2, $\rho\left(\frac{h_{n}-f}{m}\right) \rightarrow 0$. Thus $m \rho\left(\frac{h_{n}-f}{m}\right) \rightarrow 0$. Thus, the fixedpoint iteration (1.15) is weakly $\rho$-stable.

(3) Suppose that $m>1$ and that $\forall g \in D \rho(g-f)=m \rho\left(\frac{g-f}{m}\right)$. If $\lim _{n \rightarrow \infty} \varepsilon_{n}=0$, then by (3.36) and Lemma 1.2, $h_{n} \rightarrow f$. Thus, the fixed-point iteration (1.15) is $\rho$-stable.

Theorem 3.3 leads to the following corollary:

Corollary 3.3. Let $D$ be a $\rho$-closed, $\rho$-bounded and convex subset of a $\rho$-complete modular space $L_{\rho}$. Let $T: D \rightarrow P_{\rho}(D)$ be a multivalued mapping satisfying property (I), such that $P_{\rho}^{T}$ is an $m$-strong $\rho$-quasi-contractive-like mapping, satisfying contractive-like condition (3.7), where $m \in \mathbb{N}$. Suppose that $F_{\rho}(T) \neq \varnothing$. Let $f_{0} \in D$ and $\left\{f_{n}\right\} \subset D$ be defined by the implicit Noor (1.16), implicit Ishikawa (1.17), implicit Mann (1.18) iterative sequence respectively, where the sequences $\left\{\alpha_{n}\right\}_{n=0}^{\infty},\left\{\beta_{n}^{1}\right\}_{n=0}^{\infty},\left\{\beta_{n}^{2}\right\}_{n=0}^{\infty} \subset[0,1)$ are such that $\left\{\alpha_{n}\right\}_{n=0}^{\infty}$ is bounded away from 0 . Then, (1.16)-(1.18) are:

(1) relatively $\rho$-stable with respect to $T$ if $m=1$;

(2) weakly $\rho$-stable with respect to $T$ if $m>1$.

(3) $\rho$-stable with respect to $T$ if $m>1$ and $\forall g \in D \rho(g-f)=m \rho\left(\frac{g-f}{m}\right)$ where $f \in F_{\rho}(T)$ (in this case, $P_{\rho}^{T}$ is a $\rho$-quasi-contractive-like map). 
3.3.1 Numerical example. Let $M[0,1]$ be the collection of all real-valued measurable functions on $[0,1]$ and $\rho: M[0,1] \rightarrow \mathbb{R}$ a convex function modular defined by $\rho(f)=\int_{0}^{1}|f|$ $\forall f \in M[0,1]$. Let $D=\left\{f \in L_{\rho}: 0 \leq f(x) \leq 2 \forall x \in[0,1]\right\}$ be a subset of the modular function space $L_{\rho}=M[0,1]$ defined by $\rho . D$ is nonempty, closed and convex.

Define map $T: D \rightarrow P_{\rho}(D)$ by $T f=\{\delta f\}$, where $\delta=0.9$. $T$ satisfies property $(I)$, has a unique fixed point $f=0$ (since $0 \in T(0))$, and $P_{\rho}^{T}$ is a $\rho$-contraction, with $P_{\rho}^{T}(f)=\{T f\} \forall f \in D$. In fact, $P_{\rho}^{T}$ is an m-strong $\rho$-strong contraction for all $m \in \mathbb{N}$, since $\rho(g)=m \rho\left(\frac{g}{m}\right)$.

We present the results of convergence to $f=0$ of a multistep iterative sequence (1.9), an explicit multistep-SP iterative sequence (1.13) and an implicit multistep iterative sequence (1.15) using MATLAB. The parameters used are the following: $f_{0}(x)=0.5 x+0.95 \forall x \in[0,1]$, $\alpha_{n}=\frac{1}{4}+\frac{1}{n+2}, \beta_{n}^{i}=\frac{1}{n+2}$ for $i=1,2, \ldots, k-1$, where $k=11$ and $n=1,2, \ldots, 100$ (see Tables 1 and 2).

\begin{tabular}{|c|c|c|c|c|}
\hline$N$ & Explicit multistep $f_{n}(x)$ & Explicit multistep-SP $f_{n}(x)$ & Implicit multistep $f_{n}(x)$ & \\
\hline 0 & $0.5000 x+0.9500$ & $0.5000 x+0.9500$ & $0.5000 x+0.9500$ & \\
\hline 1 & $0.4583 x+0.8708$ & $0.3470 x+0.6593$ & $0.3904 x+0.7418$ & \\
\hline 16 & $0.2461 \mathrm{x}+0.4676$ & $0.0443 \mathrm{x}+0.0842$ & $0.0695 x+0.1320$ & \\
\hline 17 & $0.2383 x+0.4527$ & $0.0410 x+0.0779$ & $0.0648 x+0.1230$ & \\
\hline 24 & $0.1917 x+0.3642$ & $0.0252 x+0.0480$ & $0.0417 x+0.0792$ & \\
\hline 25 & $0.1860 x+0.3534$ & $0.0237 x+0.0450$ & $0.0394 x+0.0748$ & \\
\hline 60 & $0.0690 x+0.1311$ & $0.0043 x+0.0081$ & $0.0080 \mathrm{x}+0.0152$ & \\
\hline 61 & $0.0671 x+0.1276$ & $0.0041 x+0.0078$ & $0.0077 x+0.0146$ & \\
\hline$:$ & $\begin{array}{c}: \\
00435 x+00827\end{array}$ & $\begin{array}{c}\vdots \\
00022 x+00042\end{array}$ & $\begin{array}{c}\vdots \\
00042 x+00080\end{array}$ & \\
\hline 78 & $0.0424 \mathrm{x}+0.0805$ & $0.0021 \mathrm{x}+0.0040$ & $0.0041 x+0.0077$ & \\
\hline 79 & $0.0412 x+0.0783$ & $0.0020 x+0.0039$ & $0.0039 x+0.0075$ & \\
\hline $\begin{array}{l}\vdots \\
101\end{array}$ & $0.0229 x+0.0435$ & $0.0009 x+0.0017$ & $0.0018 x+0.0035$ & $\begin{array}{r}\text { Table } 1 . \\
\text { Convergence }\end{array}$ \\
\hline$N$ & Explicit multistep & Explicit multistep-SP & Implicit multistep & \\
\hline 0 & 1.2 . & 1.2 . & 1.2 . & \\
\hline 16 & $0 . \dot{5906}$ & $0 . \dot{1064}$ & $0 . \dot{1667}$ & \\
\hline 17 & 0.5719 & 0.0984 & 0.1554 & \\
\hline$\vdots$ & $\vdots$ & $\vdots$ & $\vdots$ & \\
\hline 24 & 0.4601 & 0.0606 & 0.1001 & \\
\hline 25 & 0.4464 & 0.0569 & 0.0945 & \\
\hline 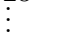 & $\vdots$ & $\vdots$ & $\vdots$ & \\
\hline 60 & 0.1656 & 0.0103 & 0.0192 & \\
\hline 61 & $0.16 \underline{11}$ & 0.0099 & 0.0184 & \\
\hline 77 & $0 . \dot{1044}$ & 0.0053 & 0.0101 & \\
\hline 78 & 0.1016 & 0.0051 & 0.0098 & \\
\hline 79 & 0.0990 & 0.0049 & 0.0094 & Table 2. \\
\hline $\begin{array}{l}\vdots \\
101\end{array}$ & $\begin{array}{c}\vdots \\
0.0550\end{array}$ & 0.0022 & 0.0044 & $\begin{array}{r}\text { Approximates } \\
\rho\left(f_{n}-f\right)\end{array}$ \\
\hline
\end{tabular}

Multistep-type construction of fixed point... 
AJMS

27,2
For this example, the explicit multistep-SP sequence seems to converge to the fixed point $f=0$ slightly faster than the implicit multistep sequence, with approximates $\rho\left(f_{n}-f\right)$ under $10^{-2}$ at $n=17$ and $n=25$ respectively, while the explicit multistep sequence is considerably slower, with $\rho\left(f_{n}-f\right)<10^{-2}$ only from $n=79$.

\section{Conclusion}

In Theorems 2.1-2.3, the fixed points of multivalued maps $T$ with a $\rho$-contractive-like associate map $P_{\rho}^{T}$ in modular spaces are successfully approximated, with supporting proofs and a numerical example, via the explicit multistep (1.9), the explicit multistep-SP (1.13) and the implicit multistep (1.15) iterative sequences. These sequences involve more steps $(k \geq 1)$ than the iterations considered in $[6,7]$.

In an attempt to prove the stability of these iterations, a new approach is used to match the convexity structure of $\rho$ : the concepts of relative $\rho$-stability (3.3) and weak $\rho$-stability (3.4) are introduced for the first time in literature, as well as the notions of $\mathrm{m}$-strong $\rho$-quasicontraction types (3.5)-(3.7), where $m \in \mathbb{N}$, which coincide with quasi-contraction types when $\rho$ is nonnegative homogeneous. Theorems 3.1-3.3 then state conditions under which schemes (1.9), (1.13) and (1.15) are $\rho$-stable, relatively $\rho$-stable and weakly $\rho$-stable, when $P_{\rho}^{T}$ is an $m$ strong $\rho$-quasi-contractive-like mapping. The proofs of this theorem are fundamentally different from those of parallel results in metric spaces as they elegantly cut out the use of triangle inequality.

\section{References}

1. Khamsi MA, Kozlowski WM. Fixed point theory in modular function spaces: Springer International Publishing 2015.

2. Khan K. Approximating fixed points of $(\lambda, \rho)$-firmly nonexpansive mappings in modular function spaces. Arab J Math. 2018; 7. doi: 10.1007/s40065-018-0204-x.

3. Khan SH, Abbas M. Approximating fixed points of multivalued Ï -nonexpansive mappings in modular function spaces. Fixed Point Theory Appl. 2014; 34: 9.

4. Khan SH, Abbas M, Ali S. Fixed point approximation of multivalued İ -quasi-nonexpansive mappings in modular function spaces. J Nonlinear Sci Appl. 2017; 10: 3168-179.

5. Kutbi MA, Latif A. Fixed points of multivalued mappings in modular function spaces. Fixed Point Theory Appl. 2009; 2009: 12.

6. Okeke GA, Bishop SA and Khan SH. Iterative approximation of fixed point of multivalued İ-quasinonexpansive mappings in modular function spaces with applications. J Fun Spaces. 2018; 2018: 9.

7. Okeke GA, Khan SH. Approximation of fixed point of multivalued $\rho$-quasi-contractive mappings in modular function spaces. Arab J Mat Sci. 2019; 26(1/2): 75-93 (accessed 3 February 2019).

8. Zamfirescu T. Fixed point theorems in metric spaces. Arch Math. 1972; 23: 292-98.

9. Osilike MO. Stability results for Ishikawa fixed point iteration procedure. Indian J Pure Appl Math. 1995/96; 26(10): 937-41.

10. Imoru CO, Olatinwo MO. On the stability of Picard and Mann iteration. Carpathian J Mat. 2003; 19: $155-60$

11. Akewe H. Approximation of fixed and common fixed points of generalized contractive-like operators. Ph.D. Thesis, Lagos, Nigeria: University of Lagos. 2010: 112.

12. Akewe H, Okeke GA, Olayiwola A. Strong convergence and stability of Kirk-multistep-type iterative schemes for contractive-type operators. Fixed Point Theory Appl. 2014 (45): 24. 
13. Berinde V. On the stability of some fixed point procedures, Buletinul Stiintific al Universitatii din Baia Mare. Seria B. Fascicola Mathematica-Informatica. 2002; XVIII(1): 7-14.

14. Berinde V. Iterative approximation of fixed points, Baia Mare: Efemeride. 2002.

15. Berinde V. On the convergence of the Ishikawa iteration in the class of quasi-contractive Multistep-type construction of fixed point... operators. Acta Math Univ Comen. 2004; LXXIII(1): 119-26.

16. Chugh R, Malik P, and Kumar V. On a new faster implicit fixed point iterative scheme in convex metric space. J Fun Spaces. 2015; 2015: 11.

17. Harder AM and Hicks TL. Stability results for fixed point iteration procedures. Math Japonica. 1988; 33(5): 693-706.

18. Ostrowski AM. The round-off stability of iterations. Zeilschrift fur Angewandte Mathemalik und Mechanik. 1967; 47: 77-81.

19. Rhoades BE. Fixed point theorems and stability results for fixed point iteration procedures. Indian J Pure Appl Math. 1990; 21: 1-9.

20. Rhoades BE. Fixed point theorems and stability results for fixed point iteration procedures II. Indian J Pure Appl Math. 1993; 24(11): 691-703.

21. Rhoades BE and Soltuz SM. The equivalence between Mann-Ishikawa iterations and multi-step iteration. Nonlinear Anal. 2004; 58: 219-28.

22. Olaleru JO and Akewe H. On the convergence of Jungck-type iterative schemes for generalized contractive-like operators. Fas Mat. 2010; 45: 87-98.

\section{Corresponding author}

Hallowed Olaoluwa can be contacted at: holaoluwa@unilag.edu.ng

For instructions on how to order reprints of this article, please visit our website: 